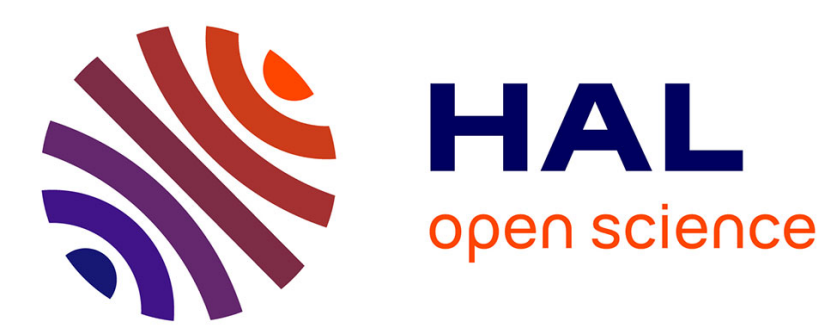

\title{
Walker Regime for Walls in Ferromagnetic Nanotubes
}

Gilles Carbou

\section{To cite this version:}

Gilles Carbou. Walker Regime for Walls in Ferromagnetic Nanotubes. Nonlinear Analysis: Real World Applications, 2018, 41, pp.642-664. 10.1016/j.nonrwa.2017.11.012 . hal-01725831

\section{HAL Id: hal-01725831 \\ https://hal.science/hal-01725831}

Submitted on 7 Mar 2018

HAL is a multi-disciplinary open access archive for the deposit and dissemination of scientific research documents, whether they are published or not. The documents may come from teaching and research institutions in France or abroad, or from public or private research centers.
L'archive ouverte pluridisciplinaire HAL, est destinée au dépôt et à la diffusion de documents scientifiques de niveau recherche, publiés ou non, émanant des établissements d'enseignement et de recherche français ou étrangers, des laboratoires publics ou privés. 


\title{
Walker Regime for Walls in Ferromagnetic Nanotubes
}

\author{
Gilles Carbou \\ CNRS / UNIV PAU \& PAYS ADOUR, \\ Laboratoire de Mathématiques et de leurs Applications de Pau, UMR CNRS 5142, \\ Avenue de l'Université - BP 1155, 64013 PAU CEDEX, FRANCE \\ email: gilles.carbou@univ-pau.fr
}

\begin{abstract}
Ferromagnetic nanotubes are proposed as an alternative to ferromagnetic nanowires for data-storage applications. In this paper, we consider a two-dimensional model for such devices and we establish the stability of moving walls in the Walker regime when the tube is subject to a small magnetic field.
\end{abstract}

$M S C: 35 \mathrm{~K} 55,35 \mathrm{Q} 60$.

Keywords: Landau-Lifschitz equation, domain walls, stability.

\section{Introduction}

Domain walls formation and propagation in ferromagnetic nanowires are intensively studied. Indeed, their possible applications for data recording (see [19]) or in nano-electronics (see [1]) are very promising. Such devices are modeled by a 1d-Landau-Lifschitz equation, and existence and stability of one-wall profiles are established (see $[10,11,12,21]$ and the references therein).

In [24], the authors propose to use ferromagnetic nanotubes instead of ferromagnetic nanowires or nano strips in order to deal with domain wall motion in the Walker regime, which is stabler and more reliable for applications. In the present work we exhibit a $2 \mathrm{~d}$-model for ferromagnetic nanotubes and we study domain wall dynamics in this model for a small applied magnetic field.

Let us recall the 3 -dimensional model for a ferromagnetic sample $\mathcal{O} \subset \mathbb{R}^{3}$. We denote by $(u \cdot v)$ the canonical scalar product of $u$ by $v$ in $\mathbb{R}^{3}$ and by $|$.$| the associated norm. The canonical basis of \mathbb{R}^{3}$ is denoted by $\left(e_{1}, e_{2}, e_{3}\right)$ and $\times$ is the usual cross product.

Ferromagnetic materials are characterized by a spontaneous magnetization described by the magnetic moment $M$ defined on $\mathbb{R}^{+} \times \mathcal{O}$ and satisfying the saturation constraint

$$
|M(\mathrm{t}, x)|=M_{s} \text { a.e., }
$$

where $M_{s}$ is constant. The magnetic moment satisfies the Landau-Lifschitz equation

$$
\frac{\partial M}{\partial \mathrm{t}}=-\gamma M \times H_{e}-\frac{\alpha \gamma}{M_{s}} M \times\left(M \times H_{e}\right),
$$

in which $\gamma>0$ is the gyromagnetic ratio, $\alpha>0$ is the damping coefficient, $H_{e}$ is the effective field given by:

$$
H_{e}=\frac{A}{\mu_{0} M_{s}^{2}} \Delta M+H_{d}(M)+H_{a p p} .
$$

Here, $A>0$ is the exchange coefficient, $\mu_{0}$ is the permeability of the vacuum, $H_{a p p}$ is the applied magnetic field, and $H_{d}(M)$ is the demagnetizing field generated by the magnetization $M$. In the quasi-stationary model, the operator $H_{d}$ is given by

$$
\left\{\begin{array}{l}
\operatorname{div}\left(H_{d}(M)+\bar{M}\right)=0 \\
\operatorname{curl} H_{d}(M)=0
\end{array}\right.
$$

where $\bar{M}$ is the extension of $M$ by zero outside $\mathcal{O}$. 
The energy associated to a configuration $M$ is given by:

$$
\mathcal{E}(M)=\frac{A}{2 M_{s}^{2}} \int_{\mathcal{O}}|\nabla M|^{2} d x+\frac{\mu_{0}}{2} \int_{\mathbb{R}^{3}}\left|H_{d}(M)\right|^{2} d x-\mu_{0} \int_{\mathcal{O}} H_{a} \cdot M d x,
$$

and we have $H_{e}=-\frac{1}{\mu_{0}} \partial_{M} \mathcal{E}$.

Existence of weak or strong solutions for (1.2) is addressed in several papers (see $[2,6,7,8,13,15$, $18,22])$.

We focus now on the case of a thin nanotube of axis $\mathbb{R} e_{1}$ with circular section. The nanotube is assimilated to the cylinder $\mathbb{R} \times \rho S^{1}=\left\{(\mathrm{x}, \mathrm{y}, \mathrm{z}) \in \mathbb{R}^{3}, \quad \mathrm{y}^{2}+\mathrm{z}^{2}=\rho^{2}\right\}$. We assume that a magnetic field $H_{a p p}$ is applied in the direction of the tube axis: $H_{a p p}=H_{a} e_{1}, H_{a} \in \mathbb{R}$. We use the twodimensional model of ferromagnetic thin film obtained in [5] and [14], in which the demagnetizing field reduces to an anisotropic local term forcing $M$ to be tangent to the thin domain. In the case of our nanotube the demagnetizing field is described by the term $-(M \cdot \mathrm{n}) \mathrm{n}$, derived from the limit demagnetizing energy $\frac{\mu_{0}}{4} \int_{\mathbb{R} \times S^{1}}|M \cdot \mathrm{n}|^{2} d \sigma$, where $\mathrm{n}$ is the unit normal vector to the cylinder surface. In cylindrical coordinates, we write $\mathrm{y}=\rho \cos y$ and $\mathrm{z}=\rho \sin y$, and we obtain the following $2 \mathrm{~d}$ model:

$$
\left\{\begin{array}{l}
M:(t, \mathrm{x}, y) \rightarrow S^{2}, \quad 2 \pi \text {-periodic in the variable } y \\
\frac{\partial M}{\partial \mathrm{t}}=-\gamma M \times h(M)-\frac{\alpha \gamma}{M_{s}} M \times(M \times h(M)) \\
h(M)=\frac{A}{\mu_{0} M_{s}^{2}} \frac{\partial^{2} M}{\partial \mathrm{x}^{2}}+\frac{A}{\mu_{0} M_{s}^{2} \rho^{2}} \frac{\partial^{2} M}{\partial y^{2}}-(M \cdot \mathrm{n}(y)) \mathrm{n}(y)+H_{a} e_{1}
\end{array}\right.
$$

where the unit normal vector $\mathrm{n}$ is given by $\mathrm{n}(y)=\left(\begin{array}{c}0 \\ \cos y \\ \sin y\end{array}\right)$.

We denote $\mathrm{n}^{\perp}(y)=\left(\begin{array}{c}0 \\ -\sin y \\ \cos y\end{array}\right)$. By the rescaling $t=\frac{\gamma A \mathrm{t}}{\mu_{0} M_{s} \rho^{2}}$ and $x=\frac{\mathrm{x}}{\rho}$, we describe $M$ in the frame $\left(e_{1}, \mathrm{n}(y), \mathrm{n}^{\perp}(y)\right)$ writing:

$M(t, \mathrm{x}, y)=M_{s}\left(\mathbf{m}_{1}\left(\frac{\gamma A \mathrm{t}}{\mu_{0} M_{s} \rho^{2}}, \frac{\mathrm{x}}{\rho}, y\right) e_{1}+\mathbf{m}_{2}\left(\frac{\gamma A \mathrm{t}}{\mu_{0} M_{s} \rho^{2}}, \frac{\mathrm{x}}{\rho}, y\right) \mathrm{n}(y)+\mathbf{m}_{3}\left(\frac{\gamma A \mathrm{t}}{\mu_{0} M_{s} \rho^{2}}, \frac{\mathrm{x}}{\rho}, y\right) \mathrm{n}^{\perp}(y)\right)$.

We obtain that $M$ satisfies (1.5) if and only if $\mathbf{m}=\left(\begin{array}{l}\mathbf{m}_{1} \\ \mathbf{m}_{2} \\ \mathbf{m}_{3}\end{array}\right)$ satisfies

$$
\left\{\begin{array}{l}
\mathbf{m}:(t, x, y) \rightarrow S^{2}, \quad 2 \pi \text {-periodic in the variable } y \\
\frac{\partial \mathbf{m}}{\partial t}=-\mathbf{m} \times \mathbf{h}(\mathbf{m})-\alpha \mathbf{m} \times(\mathbf{m} \times \mathbf{h}(\mathbf{m})) \\
\mathbf{h}(\mathbf{m})=\partial_{x x} \mathbf{m}+\partial_{y y} \mathbf{m}+2 e_{1} \times \partial_{y} \mathbf{m}+\mathbf{m}_{1} e_{1}-\kappa \mathbf{m}_{3} e_{3}+h_{a} e_{1}
\end{array}\right.
$$

where $\kappa=\frac{\mu_{0} M_{s}^{2} \rho^{2}}{A}$ and $h_{a}=\frac{\mu_{0} M_{s} \rho^{2}}{A} H_{a}$.

Remark 1.1. In our model of ferromagnetic thin layer, the demagnetizing field behaves like the planar anisotropy term $-\kappa \mathbf{m}_{3} e_{3}$. The curvature of the tube induces another anisotropic effect since the tube axis $\mathbb{R} e_{1}$ becomes an easy axis of magnetization modeled by the term $+\mathbf{m}_{1} e_{1}$ in the resulting effective field $\mathbf{h}(\mathbf{m})$. 
We deal with domain wall profiles in the Walker regime as in [24]. For a vanishing applied field $\left(h_{a}=0\right)$, we observe the formation of domains in which the magnetization is along the tube axis. One-wall configuration separating a $-e_{1}$ domain and a $+e_{1}$ domain is described by the steady state solution $\mathbf{M}_{0}$ given by

$$
\mathbf{M}_{0}(x)=\left(\begin{array}{c}
\tanh x \\
1 / \cosh x \\
0
\end{array}\right) .
$$

Furthermore, a small applied field in the $e_{1}$-direction induces wall motion. This situation is described in our model by the solution:

$$
\mathbf{M}^{h_{a}}(t, x, y)=\mathbf{R}_{\theta}\left(\mathbf{M}_{0}\left(\frac{x-c t}{\delta}\right)\right)
$$

where we denote by $\mathbf{R}_{\theta}$ the rotation matrix:

$$
\mathbf{R}_{\theta}=\left(\begin{array}{ccc}
1 & 0 & 0 \\
0 & \cos \theta & -\sin \theta \\
0 & \sin \theta & \cos \theta
\end{array}\right)
$$

and where $c, \theta$ and $\delta$ depend on $h_{a}$ as follows:

$$
h_{a}=\alpha \kappa \sin \theta \cos \theta, \quad \frac{c}{\delta}=-\alpha h_{a}-\kappa \cos \theta \sin \theta, \quad \frac{1}{\delta^{2}}=1+\kappa \sin ^{2} \theta
$$

This solution is only defined for $\left|h_{a}\right| \leq \frac{\alpha \kappa}{2}$, since $h_{a}=\frac{\alpha \kappa}{2} \sin 2 \theta$.

Remark 1.2. This kind of solution only depending on the $x$-variable is also observed in $1 d$-models of nanowires with elliptical sections (see [20] and [21]) and in Walker's 3d-model in [23].

In this paper we establish that the solution $\mathbf{M}^{h_{a}}$ is stable in the Lyapunov sense. We also prove that $\mathbf{M}^{h_{a}}$ is asymptotically stable modulo translations in the $x$-variable.

We use the following notations:

- $\Omega=\mathbb{R} \times] 0,2 \pi[$,

- $\mathbf{L}_{p}^{2}$ is the space of the measurable functions $u:(x, y) \rightarrow \mathbb{R}^{l}(l=1,2$ or 3$)$ which are $2 \pi$-periodic in $y$, and such that $u \in L^{2}\left(\Omega ; \mathbb{R}^{l}\right)$. We denote by $\langle\mid\rangle$ the associated inner product

$$
\langle u \mid v\rangle=\int_{\Omega}(u(x, y) \cdot v(x, y)) \mathrm{d} x \mathrm{~d} y
$$

and by $\|\cdot\|_{\mathbf{L}_{p}^{2}}$ the associated norm.

- $\mathbf{H}_{p}^{k}$ is the space of the measurable functions $u:(x, y) \rightarrow \mathbb{R}^{l}(l=1,2$ or 3$)$ which are $2 \pi$ periodic in $y$ and such that $u$ belongs to the Sobolev space $H^{k}\left(\Omega ; \mathbb{R}^{p}\right)$. The associated norm is denoted by $\|\cdot\|_{\mathbf{H}_{p}^{k}}$.

Our main result is the following stability theorem:

Theorem 1. There exists $h_{\max }, 0<h_{\max }<\frac{\alpha \kappa}{2}$, such that if $\left|h_{a}\right| \leq h_{\max }$, then for all $\varepsilon>0$, there exists $\eta>0$ such that if $\mathbf{m}$ satisfies (1.6) with $\left\|\mathbf{m}(0, \cdot)-\mathbf{M}^{h_{a}}(0, \cdot)\right\|_{\mathbf{H}_{p}^{2}} \leq \eta$, then:

- for all $t>0, \quad\left\|\mathbf{m}(t, \cdot)-\mathbf{M}^{h_{a}}(t, \cdot)\right\|_{\mathbf{H}_{p}^{2}} \leq \varepsilon \quad$ (stability),

- there exists $\sigma_{\infty} \in \mathbb{R}$ such that $\left\|\mathbf{m}(t, \cdot)-\mathbf{M}^{h_{a}}\left(t, \cdot-\sigma_{\infty}\right)\right\|_{\mathbf{H}_{p}^{2}}$ tends to 0 when $t$ tends to $+\infty$ (asymptotic stability modulo translations). 
To our knowledge, this work is the first one dealing with the stability of moving walls structures in dimension strictly greater than 1 . In the $3 \mathrm{~d}$-model of [4], only static walls are studied. In addition, the model in [4] is not complete since the demagnetizing field is unduly simplified in 3d. Here, the model is more convincing since the $2 \mathrm{~d}$-model for the demagnetizing field can be justified by asymptotic arguments (see [5] and [14]).

Roughly speaking, in the proof of Theorem 1, we use the techniques developed in [4] and [10]. We have to address several difficulties, some of them being specific to the nanotube case.

The first one comes from the saturation constraint (1.1), since we must consider only perturbations satisfying this constraint. To overcome this problem we use a moving frame technique as in [10] (see Section 2).

In Section 3, we prove a linear stability result. We prove that the linearization around the studied solution is non negative outside its kernel. This kernel is one-dimensional and relates to the invariance by translation of the Landau-Lifschitz type equation (1.6). The coercivity proof is specific to our $2 \mathrm{~d}$ case and is quite tricky because of the term $e_{1} \times \partial_{y} \mathbf{m}$ in the effective field in (1.6).

Theorem 1 is established in Section 4. The zero eigenvalue due to the translation invariance is responsible for a drift of the perturbation. As in $[16,17]$ and the references therein, we split the perturbations of $\mathbf{M}^{h a}$ as a translation of $\mathbf{M}^{h a}$ plus a residual term. The linear estimates of Section 3 and variational estimates yield that this remainder term tends exponentially to zero when $t$ tends to $+\infty$.

For the convenience of the reader, we postpone the technical estimates of the nonlinear terms to Section 5.

\section{Moving Frame Technique}

The magnetic applied field $h_{a}$ being fixed, we introduce $c, \delta$ and $\theta$ given by (1.10). Then we write the solution $\mathbf{m}$ of (1.6) on the form:

$$
\mathbf{m}(t, x, y)=\mathbf{R}_{\theta} \mathbf{v}\left(t, \frac{x-c t}{\delta}, y\right)
$$

where $\mathbf{v}=\left(\begin{array}{c}\mathbf{v}_{1} \\ \mathbf{v}_{2} \\ \mathbf{v}_{3}\end{array}\right)$ satisfies the saturation constraint $|\mathbf{v}|=1$, so that $\mathbf{m}$ satisfies (1.6) if and only if v satisfies

$$
\partial_{t} \mathbf{v}-\frac{c}{\delta} \partial_{x} \mathbf{v}=-\mathbf{v} \times \mathbf{H}(\mathbf{v})-\alpha \mathbf{v} \times(\mathbf{v} \times \mathbf{H}(\mathbf{v}))
$$

where

$$
\mathbf{H}(\mathbf{v})=\frac{1}{\delta^{2}} \partial_{x x} \mathbf{v}+\partial_{y y} \mathbf{v}+2 e_{1} \times \partial_{y} \mathbf{v}+\mathbf{v}_{1} e_{1}-\kappa\left(\sin \theta \mathbf{v}_{2}+\cos \theta \mathbf{v}_{3}\right)\left(\sin \theta e_{2}+\cos \theta e_{3}\right)+h_{a} e_{1} .
$$

In addition, $\mathbf{M}^{h_{a}}$ is stable for (1.6) if and only if $\mathbf{M}_{0}$ is stable for (2.11).

Therefore in order to establish Theorem 1, we aim to prove that if $\left\|\mathbf{v}(0, \cdot)-\mathbf{M}_{0}(\cdot)\right\|_{\mathbf{H}_{p}^{2}}$ is small enough, then $\left\|\mathbf{v}(t, \cdot)-\mathbf{M}_{0}(\cdot)\right\|_{\mathbf{H}_{p}^{2}}$ remains small for all $t$ and there exists $\sigma_{\infty}$ such that $\left\|\mathbf{v}(t, \cdot)-\mathbf{M}_{0}\left(\cdot-\sigma_{\infty}\right)\right\|_{\mathbf{H}_{p}^{2}}$ tends to zero when $t$ tends to $+\infty$.

In order to deal with perturbations $\mathbf{v}$ of $\mathbf{M}_{0}$ satisfying the saturation constraint $|\mathbf{v}|=1$, we use the mobile frame technique developed in [10]. We introduce $\mathbf{M}_{1}$ and $\mathbf{M}_{2}$ defined by

$$
\mathbf{M}_{1}(x)=\left(\begin{array}{c}
-1 / \cosh x \\
\tanh x \\
0
\end{array}\right) \text { and } \mathbf{M}_{2}=\left(\begin{array}{l}
0 \\
0 \\
1
\end{array}\right)
$$

and we write $\mathbf{v}$ on the form

$$
\mathbf{v}(t, x, y)=\mathbf{M}_{0}(x)+r_{1}(t, x, y) \mathbf{M}_{1}(x)+r_{2}(t, x, y) \mathbf{M}_{2}+\mu(r(t, x, y)) \mathbf{M}_{0}(x),
$$


where the new unknown $r:(t, x, y) \mapsto\left(\begin{array}{c}r_{1}(t, x, y) \\ r_{2}(t, x, y)\end{array}\right) \in \mathbb{R}^{2}$ is $2 \pi$-periodic in the $y$-variable and where $\mu$ is chosen so that the saturation constraint in always satisfied:

$$
\mu(\xi)=\sqrt{1-\left(\xi_{1}\right)^{2}-\left(\xi_{2}\right)^{2}}-1 .
$$

Plugging this formulation in (2.11) and taking the scalar product with $\mathbf{M}_{1}$ and $\mathbf{M}_{2}$, we obtain that $\mathbf{v}$ satisfies (2.11) if and only if $r$ satisfies:

$$
\frac{\partial r}{\partial t}=\left(\begin{array}{cc}
-\alpha & -1 \\
1 & -\alpha
\end{array}\right) \mathcal{M} r-h_{a} \mathcal{L} r+\mathcal{F}(r)
$$

where the linear operators $\mathcal{M}$ and $\mathcal{L}$ are defined by

$$
\mathcal{M}=\left(\begin{array}{cc}
\frac{1}{\delta^{2}} L-\partial_{y y} & 2 \tanh x \partial_{y} \\
-2 \tanh x \partial_{y} & \frac{1}{\delta^{2}} L-\partial_{y y}+\kappa \cos 2 \theta
\end{array}\right), \quad \text { with } L=-\partial_{x x}+\left(2 \tanh ^{2}-1\right),
$$

and

$$
\mathcal{L} r=\left(\alpha+\frac{1}{\alpha}\right) \ell r+2 \tanh x r_{2}\left(\begin{array}{c}
1 \\
-\frac{1}{\alpha}
\end{array}\right), \quad \text { with } \ell=\partial_{x}+\tanh x
$$

and where the term $\mathcal{F}: \mathbf{H}_{p}^{2} \rightarrow \mathbf{L}_{p}^{2}$ is the non linear contribution (that is $\left.\partial_{r} \mathcal{F}(0)=0\right)$. For the convenience of the reader, the expression of $\mathcal{F}$ is postponed to Section 5.

\section{Linear Stability}

In this part, we study the stability of the zero solution for the linearization of (2.12):

$$
\frac{\partial v}{\partial t}=\left(\begin{array}{cc}
-\alpha & -1 \\
1 & -\alpha
\end{array}\right) \mathcal{M} v-h_{a} \mathcal{L} v
$$

The linear operator $L$ in (2.13) appears in several stability proofs concerning one-dimensional models of nanowires (see $[9,10,11,12])$. It also appears in [4] for the $3 \mathrm{~d}$ case. We recall the properties of this operator (see [12] for the proofs):

- $L$ is self-adjoint with domain $H^{2}(\mathbb{R})$,

- we can factorize $L$ on the form $L=\ell^{*} \circ \ell$ with $\ell=\partial_{x}+\tanh x$, so that $L$ is positive. In addition,

$$
\int_{\mathbb{R}} u \cdot L u=\left\|L^{\frac{1}{2}} u\right\|_{L^{2}(\mathbb{R})}^{2}=\|\ell u\|_{L^{2}(\mathbb{R})}^{2},
$$

- Ker $L=\mathbb{R} \frac{1}{\cosh x}$, and the essential spectrum of $L$ is $[1,+\infty[$,

- 0 in the only eigenvalue of $L$,

- on $(\operatorname{Ker} L)^{\perp}, L \geq 1$, so that if $\int_{\mathbb{R}} \frac{u(x)}{\cosh x} \mathrm{~d} x=0$, then

$$
\|u\|_{L^{2}(\mathbb{R})} \leq\|\ell u\|_{L^{2}(\mathbb{R})} \leq\|L u\|_{L^{2}(\mathbb{R})}
$$

We introduce the following notations: 
- $\mathbf{L}_{p}^{2, \perp}=\left\{w=\left(\begin{array}{l}w_{1} \\ w_{2}\end{array}\right) \in \mathbf{L}_{p}^{2}, \quad\right.$ such that $\left.\int_{\mathbb{R} \times] 0,2 \pi[} w_{1}(t, x, y) \frac{1}{\cosh x} \mathrm{~d} x \mathrm{~d} y=0\right\}$,

- $\mathbf{H}_{p}^{k, \perp}=\mathbf{H}_{p}^{k} \cap \mathbf{L}_{p}^{2, \perp}$.

The properties of $\mathcal{M}$ are summarized in the following:

Proposition 3.1. For all $\theta \in]-\frac{\pi}{4}, \frac{\pi}{4}\left[, \mathcal{M}\right.$ is a self-adjoint positive operator for the $\mathbf{L}_{p}^{2}$ inner product with domain $\mathcal{D}(\mathcal{M})=\mathbf{H}_{p}^{2}$, and $\operatorname{Ker} \mathcal{M}=\mathbb{R} \frac{1}{\cosh x}\left(\begin{array}{l}1 \\ 0\end{array}\right)$.

In addition, $\mathcal{M}$ is non negative on $(\operatorname{Ker} \mathcal{M})^{\perp}=\mathbf{H}_{p}^{2, \perp}$, and for all fixed $\theta_{\max } \in\left[0, \frac{\pi}{4}[\right.$, there exist constants $\alpha_{1}>0, \alpha_{2}>0$ and $\alpha_{3}>0$ (depending on $\theta_{\max }$ ) such that for all $\left.\theta \in\right]-\theta_{\max }, \theta_{\max }[$,

- for $k \in\{1,2\}$, for all $w \in \mathbf{H}_{p}^{k+1, \perp}, \alpha_{1}\left\|\mathcal{M}^{\frac{k}{2}} w\right\|_{\mathbf{L}_{p}^{2}} \leq\left\|\mathcal{M}^{\frac{k+1}{2}} w\right\|_{\mathbf{L}_{p}^{2}}$,

- for $k \in\{1,2,3\}$, for all $w \in \mathbf{H}_{p}^{k, \perp}, \quad \alpha_{2}\|w\|_{\mathbf{H}_{p}^{k}} \leq\left\|\mathcal{M}^{\frac{k}{2}} w\right\|_{\mathbf{L}_{p}^{2}} \leq \alpha_{3}\|w\|_{\mathbf{H}_{p}^{k}}$.

As a corollary of Proposition 3.1, we obtain the following Theorem:

Theorem 2. There exists $h_{\max }^{l}$ with $0<h_{\max }^{l}<\frac{\alpha \kappa}{2}$, such that for all $h_{a}$ with $\left|h_{a}\right| \leq h_{\max }^{l}$, the zero solution is stable for Equation (3.15). More precisely, for all $\varepsilon>0$, there exists $\eta>0$ such that for all $v_{0} \in \mathbf{H}_{p}^{1}$, if $\left\|v_{0}\right\|_{\mathbf{H}_{p}^{1}} \leq \eta$, then the solution $v$ of (3.15) with initial data $v_{0}$ satisfies

$$
\forall t>0, \quad\|v(t, \cdot)\|_{\mathbf{H}_{p}^{1}} \leq \varepsilon .
$$

In addition, when $t$ tends to $+\infty, v(t, \cdot)$ tends in $\mathbf{H}_{p}^{1}$ to a limit of the form $\frac{\sigma_{\infty}}{\cosh x}\left(\begin{array}{l}1 \\ 0\end{array}\right)$, where $\sigma_{\infty} \in \mathbb{R}$.

Remark 3.1. We prove the linear stability in $\mathbf{H}_{p}^{1}$. We could also prove the same result in $\mathbf{H}_{p}^{2}$. For the nonlinear stability, we need $\mathbf{H}_{p}^{2}$ estimates to control the nonlinear terms. Theorem 2 is proved in Section 3.2

\subsection{Proof of Proposition 3.1}

We first establish the following Lemma:

Lemma 3.1. There exists $c_{1}>0$ and $c_{2}>0$ such that for all $w \in \mathbf{H}_{p}^{1, \perp}$,

$$
c_{1}\|w\|_{\mathbf{H}_{p}^{1}} \leq\left(\left\|\ell w_{1}\right\|_{\mathbf{L}_{p}^{2}}^{2}+\left\|\partial_{y} w_{1}\right\|_{\mathbf{L}_{p}^{2}}^{2}+\left\|\ell w_{2}\right\|_{\mathbf{L}_{p}^{2}}^{2}+\left\|\partial_{y} w_{2}\right\|_{\mathbf{L}_{p}^{2}}^{2}+\left\|w_{2}\right\|_{\mathbf{L}_{p}^{2}}^{2}\right)^{\frac{1}{2}} \leq c_{2}\|w\|_{\mathbf{H}_{p}^{1}} .
$$

Proof. We recall that $\ell=\partial_{x}+\tanh x$, so that the existence of $c_{2}$ is straightforward.

We have

$$
\left\|\partial_{x} w_{2}\right\|_{\mathbf{L}_{p}^{2}} \leq\left\|\ell w_{2}-\tanh x w_{2}\right\|_{\mathbf{L}_{p}^{2}} \leq\left\|\ell w_{2}\right\|_{\mathbf{L}_{p}^{2}}+\left\|\tanh x w_{2}\right\|_{\mathbf{L}_{p}^{2}}
$$

thus there exists $K$ such that

$$
\left\|w_{2}\right\|_{\mathbf{H}_{p}^{1}} \leq K\left(\left\|\ell w_{2}\right\|_{\mathbf{L}_{p}^{2}}+\left\|\partial_{y} w_{2}\right\|_{\mathbf{L}_{p}^{2}}+\left\|w_{2}\right\|_{\mathbf{L}_{p}^{2}}\right) .
$$

Concerning $w_{1}$, we first recall that for all $u \in H^{1}(\mathbb{R})$, we have

$$
\int_{\mathbb{R}} u(x) \frac{1}{\cosh x} \mathrm{~d} x=0 \quad \Longrightarrow \quad\|u\|_{L^{2}(\mathbb{R})} \leq\|\ell u\|_{L^{2}(\mathbb{R})} \quad \text { (see (3.16)). }
$$

We define $\tau$ by

$$
\tau(y)=\int_{x \in \mathbb{R}} \frac{1}{2 \cosh x} w_{1}(x, y) \mathrm{d} x .
$$


We split $w_{1}$ as:

$$
w_{1}(x, y)=W_{1}(x, y)+\frac{\tau(y)}{\cosh x} \quad \text { with } \forall y \in \mathbb{R}, \quad \int_{x \in \mathbb{R}} W_{1}(x, y) \frac{1}{\cosh x} \mathrm{~d} x=0 .
$$

For a fixed $y, x \mapsto W_{1}(x, y)$ is in $\left(\frac{1}{\cosh x}\right)^{\perp}$ (for the $L^{2}(\mathbb{R})$-inner product), so

$$
\left\|W_{1}(\cdot, y)\right\|_{L^{2}(\mathbb{R})} \leq\left\|\ell W_{1}(\cdot, y)\right\|_{L^{2}(\mathbb{R})},
$$

and by integration in the variable $y \in[0,2 \pi]$, using that $\ell \frac{1}{\cosh x}=0$, we obtain that

$$
\left\|W_{1}\right\|_{\mathbf{L}_{p}^{2}} \leq\left\|\ell w_{1}\right\|_{\mathbf{L}_{p}^{2}} .
$$

By the orthogonality condition in (3.18) we remark that $\left\langle W_{1} \mid \frac{1}{\cosh x}\right\rangle=0$ so

$$
\left\|w_{1}\right\|_{\mathbf{L}_{p}^{2}}^{2}=\left\|W_{1}\right\|_{\mathbf{L}_{p}^{2}}^{2}+\left\|\frac{\tau(y)}{\cosh x}\right\|_{\mathbf{L}_{p}^{2}}^{2}
$$

We have:

$$
\left\|\frac{\tau(y)}{\cosh x}\right\|_{\mathbf{L}_{p}^{2}}^{2}=\int_{x \in \mathbb{R}} \int_{y \in] 0,2 \pi[}|\tau(y)|^{2} \frac{1}{\cosh ^{2} x} \mathrm{~d} x \mathrm{~d} y=2\|\tau\|_{L^{2}([0,2 \pi])}^{2} .
$$

Since $w \in \mathbf{H}_{p}^{1, \perp}$, we have $\left\langle w_{1} \mid \frac{1}{\cosh x}\right\rangle=0$ thus

$$
\int_{] 0,2 \pi[} \tau(y) \mathrm{d} y=0
$$

Hence by Poincaré-Wirtinger inequality, we have

$$
\|\tau\|_{L^{2}(] 0,2 \pi[)} \leq\left\|\partial_{y} \tau\right\|_{L^{2}(] 0,2 \pi[)} .
$$

Therefore,

$$
\begin{aligned}
&\left\|w_{1}\right\|_{\mathbf{L}_{p}^{2}}^{2}=\left\|\ell w_{1}\right\|_{\mathbf{L}_{p}^{2}}^{2}+2 \int_{] 0,2 \pi[}\left|\partial_{y} \tau\right|^{2} \mathrm{~d} y \\
& \leq\left\|\ell w_{1}\right\|_{\mathbf{L}_{p}^{2}}^{2}+2 \int_{] 0,2 \pi[}\left|\int_{\mathbb{R}} \frac{1}{2 \cosh x} \partial_{y} w_{1}(x, y) \mathrm{d} x\right|^{2} \mathrm{~d} y, \\
& \leq\left\|\ell w_{1}\right\|_{\mathbf{L}_{p}^{2}}^{2}+2 \int_{] 0,2 \pi[}\left(\int_{\mathbb{R}} \frac{1}{4 \cosh ^{2} x} \mathrm{~d} x\right)\left(\int_{\mathbb{R}}\left|\partial_{y} w_{1}(x, y)\right|^{2} \mathrm{~d} x\right) \mathrm{d} y \\
& \quad \text { by Cauchy-Schwarz inequality, }
\end{aligned}
$$

thus

Therefore

$$
\left\|w_{1}\right\|_{\mathbf{L}_{p}^{2}}^{2} \leq\left\|\ell w_{1}\right\|_{\mathbf{L}_{p}^{2}}^{2}+\left\|\partial_{y} w_{1}\right\|_{\mathbf{L}_{p}^{2}}^{2}
$$

$$
\begin{aligned}
\left\|w_{1}\right\|_{\mathbf{H}_{p}^{1}} & =\left\|w_{1}\right\|_{\mathbf{L}_{p}^{2}}+\left\|\partial_{x} w_{1}\right\|_{\mathbf{L}_{p}^{2}}+\left\|\partial_{y} w_{1}\right\|_{\mathbf{L}_{p}^{2}}, \\
& \leq\left\|w_{1}\right\|_{\mathbf{L}_{p}^{2}}+\left\|\ell w_{1}\right\|_{\mathbf{L}_{p}^{2}}+\left\|\tanh x w_{1}\right\|_{\mathbf{L}_{p}^{2}}+\left\|\partial_{y} w_{1}\right\|_{\mathbf{L}_{p}^{2}}, \\
& \leq 3\left(\left\|\ell w_{1}\right\|_{\mathbf{L}_{p}^{2}}+\left\|\partial_{y} w_{1}\right\|_{\mathbf{L}_{p}^{2}}\right) \quad \text { using (3.19). }
\end{aligned}
$$

Coupling (3.17) and (3.20) we obtain the existence of $c_{1}$. This concludes the proof of Lemma 3.1.

In order to establish that $\mathcal{M}$ is non negative, we prove the following: 
Lemma 3.2. There exists $c>0$ such that for all $\theta \in]-\pi / 4, \pi / 4\left[\right.$, for all $w \in \mathbf{H}_{p}^{2, \perp}$, we have

$$
\langle\mathcal{M} w \mid w\rangle \geq c \cos 2 \theta\|w\|_{\mathbf{H}_{p}^{1}}^{2} .
$$

Proof. We recall that we denote $\Omega=\mathbb{R} \times[0,2 \pi]$. We have

$$
\begin{aligned}
\langle\mathcal{M} w \mid w\rangle= & \frac{1}{\delta^{2}}\left\langle L w_{1} \mid w_{1}\right\rangle+\left\|\partial_{y} w_{1}\right\|_{\mathbf{L}_{p}^{2}}^{2}+\frac{1}{\delta^{2}}\left\langle L w_{2} \mid w_{2}\right\rangle+\left\|\partial_{y} w_{2}\right\|_{\mathbf{L}_{p}^{2}} \\
& +\kappa \cos 2 \theta\left\|w_{2}\right\|_{\mathbf{L}_{p}^{2}}^{2}-2 \int_{\Omega} \tanh x w_{2} \partial_{y} w_{1}+2 \int_{\Omega} \tanh x w_{1} \partial_{y} w_{2} .
\end{aligned}
$$

We estimate the last two integrals. Let $\nu, 0<\nu \leq 1$. We have:

$$
\begin{aligned}
-2 \int_{\Omega} \tanh x w_{2} \partial_{y} w_{1} & +2 \int_{\Omega} \tanh x w_{1} \partial_{y} w_{2}=-4 \nu \int_{\Omega} \tanh x w_{2} \partial_{y} w_{1} \\
& -2(1-\nu) \int_{\Omega} \tanh x w_{2} \partial_{y} w_{1}+2(1-\nu) \int_{\Omega} \tanh x w_{1} \partial_{y} w_{2} .
\end{aligned}
$$

Since $\int_{\Omega} \partial_{x} w_{2} \partial_{y} w_{1}-\int_{\Omega} \partial_{x} w_{1} \partial_{y} w_{2}=0$, we have

$$
-\int_{\Omega} \tanh x w_{2} \partial_{y} w_{1}+\int_{\Omega} \tanh x w_{1} \partial_{y} w_{2}=-\int_{\Omega} \ell w_{2} \partial_{y} w_{1}+\int_{\Omega} \ell w_{1} \partial_{y} w_{2}
$$

(we recall that $\ell w_{i}=\partial_{x} w_{i}+\tanh x w_{i}$ ). Therefore

$$
\begin{aligned}
-2 \int_{\Omega} \tanh x w_{2} \partial_{y} w_{1}+2 \int_{\Omega} \tanh x w_{1} \partial_{y} w_{2}= & -4 \nu \int_{\Omega} \tanh x w_{2} \partial_{y} w_{1} \\
& -2(1-\nu) \int_{\Omega}\left(\ell w_{2} \partial_{y} w_{1}-\ell w_{1} \partial_{y} w_{2}\right) .
\end{aligned}
$$

Hence

$$
\begin{aligned}
\mid-2 \int_{\Omega} \tanh x w_{2} \partial_{y} w_{1}+ & 2 \int_{\Omega} \tanh x w_{1} \partial_{y} w_{2} \mid \leq 4 \nu\left\|\partial_{y} w_{1}\right\|_{\mathbf{L}_{p}^{2}}\left\|w_{2}\right\|_{\mathbf{L}_{p}^{2}} \\
& +2(1-\nu)\left\|\ell w_{1}\right\|_{\mathbf{L}_{p}^{2}}\left\|\partial_{y} w_{2}\right\|_{\mathbf{L}_{p}^{2}}+2(1-\nu)\left\|\ell w_{2}\right\|_{\mathbf{L}_{p}^{2}}\left\|\partial_{y} w_{1}\right\|_{\mathbf{L}_{p}^{2}}, \\
\leq & \frac{\nu}{2}\left\|\partial_{y} w_{1}\right\|_{\mathbf{L}_{p}^{2}}^{2}+8 \nu\left\|w_{2}\right\|_{\mathbf{L}_{p}^{2}}^{2}+(1-\nu)\left\|\ell w_{1}\right\|_{\mathbf{L}_{p}^{2}}^{2}+(1-\nu)\left\|\partial_{y} w_{2}\right\|_{\mathbf{L}_{p}^{2}}^{2} \\
& +(1-\nu)\left\|\ell w_{2}\right\|_{\mathbf{L}_{p}^{2}}^{2}+(1-\nu)\left\|\partial_{y} w_{1}\right\|_{\mathbf{L}_{p}^{2}}^{2}, \\
\leq & (1-\nu)\left\|\ell w_{1}\right\|_{\mathbf{L}_{p}^{2}}^{2}+\left(1-\frac{\nu}{2}\right)\left\|\partial_{y} w_{1}\right\|_{\mathbf{L}_{p}^{2}}^{2}+(1-\nu)\left\|\partial_{y} w_{2}\right\|_{\mathbf{L}_{p}^{2}}^{2} \\
& +(1-\nu)\left\|\ell w_{2}\right\|_{\mathbf{L}_{p}^{2}}^{2}+8 \nu\left\|w_{2}\right\|_{\mathbf{L}_{p}^{2}}^{2} .
\end{aligned}
$$

So, using this estimate in $(3.21)$, since $\left\langle L w_{i} \mid w_{i}\right\rangle=\left\|\ell w_{i}\right\|_{\mathbf{L}_{p}^{2}}^{2}$, since $\frac{1}{\delta^{2}}-1=\kappa \sin ^{2} \theta$, we have

$$
\begin{aligned}
\langle\mathcal{M} w \mid w\rangle \geq & \left(\kappa \sin ^{2} \theta+\nu\right)\left\|\ell w_{1}\right\|_{\mathbf{L}_{p}^{2}}^{2}+\frac{\nu}{2}\left\|\partial_{y} w_{1}\right\|_{\mathbf{L}_{p}^{2}}^{2}+\left(\kappa \sin ^{2} \theta+\nu\right)\left\|\ell w_{2}\right\|_{\mathbf{L}_{p}^{2}}^{2} \\
& +\nu\left\|\partial_{y} w_{2}\right\|_{\mathbf{L}_{p}^{2}}^{2}+(\kappa \cos 2 \theta-8 \nu)\left\|w_{2}\right\|_{\mathbf{L}_{p}^{2}}^{2} .
\end{aligned}
$$


We take $\nu=\nu(\theta):=\inf \left\{1, \frac{\kappa \cos 2 \theta}{16}\right\}$ and we obtain that

$$
\langle\mathcal{M} w \mid w\rangle \geq \frac{\nu(\theta)}{2}\left(\left\|\ell w_{1}\right\|_{\mathbf{L}_{p}^{2}}^{2}+\left\|\partial_{y} w_{1}\right\|_{\mathbf{L}_{p}^{2}}^{2}+\left\|\ell w_{2}\right\|_{\mathbf{L}_{p}^{2}}^{2}+\left\|\partial_{y} w_{2}\right\|_{\mathbf{L}_{p}^{2}}^{2}+\left\|w_{2}\right\|_{\mathbf{L}_{p}^{2}}^{2}\right) .
$$

Using Lemma 3.1 we obtain:

$$
\langle\mathcal{M} w \mid w\rangle \geq \frac{\left(c_{1}\right)^{2}}{2} \nu(\theta)\|w\|_{\mathbf{H}_{p}^{1}}^{2} .
$$

We remark now that there exists $c>0$ such that for all $\theta \in]-\pi / 4, \pi / 4$,

$$
\frac{\left(c_{1}\right)^{2}}{2} \nu(\theta) \geq c \cos 2 \theta \text {. }
$$

This concludes the proof of Lemma 3.2.

Proof of Proposition 3.1. For all $\theta \in]-\theta_{\max }, \theta_{\max }\left[, \cos 2 \theta \geq \cos 2 \theta_{\max }\right.$. We set

$$
\alpha_{1}=\left(c \cos 2 \theta_{\max }\right)^{\frac{1}{2}}
$$

(see Lemma 3.2) and we have, by density of $\mathbf{H}_{p}^{2, \perp}$ in $\mathbf{H}_{p}^{1, \perp}$, that

$$
\forall w \in \mathbf{H}^{1, \perp}, \quad \alpha_{1}\|w\|_{\mathbf{H}_{p}^{1}} \leq\left\|\mathcal{M}^{\frac{1}{2}} w\right\|_{\mathbf{L}_{p}^{2}},
$$

thus

$$
\forall w \in \mathbf{H}^{1, \perp}, \quad \alpha_{1}\|w\|_{\mathbf{L}_{p}^{2}} \leq\left\|\mathcal{M}^{\frac{1}{2}} w\right\|_{\mathbf{L}_{p}^{2}} .
$$

In addition,

$$
\alpha_{1}\left\|\mathcal{M}^{\frac{1}{2}} w\right\|_{\mathbf{L}_{p}^{2}}^{2}=\alpha_{1}\langle w \mid \mathcal{M} w\rangle \leq \alpha_{3}\|w\|_{\mathbf{L}_{p}^{2}}\|\mathcal{M} w\|_{\mathbf{L}_{p}^{2}} \leq\left\|\mathcal{M}^{\frac{1}{2}} w\right\|_{\mathbf{L}_{p}^{2}}\|\mathcal{M} w\|_{\mathbf{L}_{p}^{2}} .
$$

We obtain then that

$$
\alpha_{1}\left\|\mathcal{M}^{\frac{1}{2}} w\right\|_{\mathbf{L}_{p}^{2}} \leq\|\mathcal{M} w\|_{\mathbf{L}_{p}^{2}} .
$$

If $w \in \mathbf{H}_{p}^{3, \perp}$, then $\mathcal{M} w \in \mathbf{H}_{p}^{1, \perp}$. Therefore, applying (3.23) replacing $w$ by $\mathcal{M} w$, we obtain that for $w \in \mathbf{H}_{p}^{3, \perp}$,

$$
\alpha_{1}\|\mathcal{M} w\|_{\mathbf{L}_{p}^{2}} \leq\left\|\mathcal{M}^{\frac{1}{2}} w\right\|_{\mathbf{L}_{p}^{2}} .
$$

The existence of $\alpha_{3}$ is straightforward, since $\mathcal{M}$ is an order-two operator.

Concerning $\alpha_{2}$, we first remark that from Proposition 3.2 we have:

$$
\alpha_{1}\|w\|_{\mathbf{H}_{p}^{1}} \leq\left\|\mathcal{M}^{\frac{1}{2}} w\right\|_{\mathbf{L}_{p}^{2}} .
$$

In addition, writing $\Delta_{\delta}=\frac{1}{\delta^{2}} \partial_{x x}+\partial_{y y}$, we have

$$
\mathcal{M} w=-\Delta_{\delta} w+\mathcal{A}(w)
$$

where $\mathcal{A}(w)=2 \tanh x\left(\begin{array}{c}\partial_{y} w_{2} \\ -\partial_{y} w_{1}\end{array}\right)+\frac{1}{\delta^{2}}\left(1-2 \tanh ^{2} x\right) w+\kappa \cos 2 \theta\left(\begin{array}{c}0 \\ w_{2}\end{array}\right)$.

So

$$
\begin{aligned}
\left\|\Delta_{\delta} w\right\|_{\mathbf{L}_{p}^{2}} & \leq\|\mathcal{M} w\|_{\mathbf{L}_{p}^{2}}+\|\mathcal{A} w\|_{\mathbf{L}_{p}^{2}} \\
& \leq\|\mathcal{M} w\|_{\mathbf{L}_{p}^{2}}+c\|w\|_{\mathbf{H}_{p}^{1}} \text { since } \mathcal{A} \text { is an order-one operator, } \\
& \leq\|\mathcal{M} w\|_{\mathbf{L}_{p}^{2}}+\frac{c}{\alpha_{1}}\left\|\mathcal{M}^{\frac{1}{2}} w\right\|_{\mathbf{L}_{p}^{2}} \text { with }(3.22) \\
& \leq\left(1+\frac{c}{\left(\alpha_{1}\right)^{2}}\right)\|\mathcal{M} w\|_{\mathbf{L}_{p}^{2}}
\end{aligned}
$$


Since $\left\|\Delta_{\delta} w\right\|_{\mathbf{L}_{p}^{2}}^{2}=\frac{1}{\delta^{4}}\left\|\partial_{x x} w\right\|_{\mathbf{L}_{p}^{2}}^{2}+2 \frac{1}{\delta^{2}}\left\|\partial_{x y} w\right\|_{\mathbf{L}_{p}^{2}}^{2}+\left\|\partial_{y y} w\right\|_{\mathbf{L}_{p}^{2}}^{2}$ with $1 \leq \frac{1}{\delta^{2}} \leq 2$, we obtain that there exists a constant $k$ independent of $\theta$ and $w$ such that

$$
\left\|\partial_{x x} w\right\|_{\mathbf{L}_{p}^{2}}+\left\|\partial_{x y} w\right\|_{\mathbf{L}_{p}^{2}}+\left\|\partial_{y y} w\right\|_{\mathbf{L}_{p}^{2}} \leq k\|\mathcal{M} w\|_{\mathbf{L}_{p}^{2}} .
$$

Since we already know that $\|w\|_{\mathbf{H}_{p}^{1}} \leq \frac{1}{\alpha_{1}}\left\|\mathcal{M}^{\frac{1}{2}} w\right\|_{\mathbf{L}_{p}^{2}} \leq \frac{1}{\left(\alpha_{1}\right)^{2}}\|\mathcal{M} w\|_{\mathbf{L}_{p}^{2}}$, we obtain that there exists a constant $a_{2}$ such that:

$$
\forall \theta \in\left[-\theta_{\max }, \theta_{\max }\right], \forall w \in \mathbf{H}_{p}^{2, \perp}, \quad a_{2}\|w\|_{\mathbf{H}_{p}^{2}} \leq\|\mathcal{M} w\|_{\mathbf{L}_{p}^{2}} .
$$

Concerning the $H^{3}$ estimate, we remark that

$$
\begin{aligned}
\left\|\mathcal{M}^{\frac{3}{2}} w\right\|_{\mathbf{L}_{p}^{2}}^{2} & =\left\langle\mathcal{M}^{2} w \mid \mathcal{M} w\right\rangle \\
& =\left\langle\Delta_{\delta}^{2} w \mid \Delta_{\delta} w\right\rangle+\left\langle\Delta_{\delta} w \mid \Delta_{\delta} \mathcal{A} w+\mathcal{A} \Delta_{\delta} w+\mathcal{A}^{2} w\right\rangle
\end{aligned}
$$

So

$$
\begin{aligned}
\frac{1}{\delta^{2}}\left\|\partial_{x} \Delta_{\delta} w\right\|_{\mathbf{L}_{p}^{2}}^{2}+\left\|\partial_{y} \Delta_{\delta} w\right\|_{\mathbf{L}_{p}^{2}}^{2} & \leq\left\|\mathcal{M}^{\frac{3}{2}} w\right\|_{\mathbf{L}_{p}^{2}}^{2}+\left|\left\langle\Delta_{\delta} w \mid \Delta_{\delta} \mathcal{A} w+\mathcal{A} \Delta_{\delta} w+\mathcal{A}^{2} w\right\rangle\right|, \\
& \leq\left\|\mathcal{M}^{\frac{3}{2}} w\right\|_{\mathbf{L}_{p}^{2}}^{2}+\left\|\Delta_{\delta} w\right\|_{\mathbf{L}_{p}^{2}}\left\|\Delta_{\delta} \mathcal{A} w+\mathcal{A} \Delta_{\delta} w+\mathcal{A}^{2} w\right\|_{\mathbf{L}_{p}^{2}}, \\
& \leq\left\|\mathcal{M}^{\frac{3}{2}} w\right\|_{\mathbf{L}_{p}^{2}}^{2}+c\|w\|_{\mathbf{H}_{p}^{2}}\|w\|_{\mathbf{H}_{p}^{3}} .
\end{aligned}
$$

We remark that there exists $k_{1}>0$ such that

$$
\|w\|_{\mathbf{H}_{p}^{3}} \leq k_{1}\left(\frac{1}{\delta^{2}}\left\|\partial_{x} \Delta_{\delta} w\right\|_{\mathbf{L}_{p}^{2}}^{2}+\left\|\partial_{y} \Delta_{\delta} w\right\|_{\mathbf{L}_{p}^{2}}^{2}\right)^{\frac{1}{2}}+\|w\|_{\mathbf{H}_{p}^{2}}
$$

so

$$
\begin{aligned}
\frac{1}{\delta^{2}}\left\|\partial_{x} \Delta_{\delta} w\right\|_{\mathbf{L}_{p}^{2}}^{2}+\left\|\partial_{y} \Delta_{\delta} w\right\|_{\mathbf{L}_{p}^{2}}^{2} \leq & \left\|\mathcal{M}^{\frac{3}{2}} w\right\|_{\mathbf{L}_{p}^{2}}^{2}+c k_{1}\|w\|_{\mathbf{H}_{p}^{2}}\left(\frac{1}{\delta^{2}}\left\|\partial_{x} \Delta_{\delta} w\right\|_{\mathbf{L}_{p}^{2}}^{2}+\left\|\partial_{y} \Delta_{\delta} w\right\|_{\mathbf{L}_{p}^{2}}^{2}\right)^{\frac{1}{2}} \\
& +c\|w\|_{\mathbf{H}_{p}^{2}}^{2}, \\
\leq & \left(c+\frac{c^{2}\left(k_{1}\right)^{2}}{2}\right)\|w\|_{\mathbf{H}_{p}^{2}}^{2}+\left\|\mathcal{M}^{\frac{3}{2}} w\right\|_{\mathbf{L}_{p}^{2}}^{2} \\
& +\frac{1}{2}\left(\frac{1}{\delta^{2}}\left\|\partial_{x} \Delta_{\delta} w\right\|_{\mathbf{L}_{p}^{2}}^{2}+\left\|\partial_{y} \Delta_{\delta} w\right\|_{\mathbf{L}_{p}^{2}}^{2}\right) \text { by Young inequality. }
\end{aligned}
$$

Therefore we obtain that

$$
\frac{1}{\delta^{2}}\left\|\partial_{x} \Delta_{\delta} w\right\|_{\mathbf{L}_{p}^{2}}^{2}+\left\|\partial_{y} \Delta_{\delta} w\right\|_{\mathbf{L}_{p}^{2}}^{2} \leq 2\left\|\mathcal{M}^{\frac{3}{2}} w\right\|_{\mathbf{L}_{p}^{2}}^{2}+2\left(c+\frac{c^{2}\left(k_{1}\right)^{2}}{2}\right)\|w\|_{\mathbf{H}_{p}^{2}}^{2} .
$$

Using (3.25), (3.24) and the previous $H^{2}$-estimates, we obtain that there exists $a_{3}>0$ such that

$$
a_{3}\|w\|_{\mathbf{H}_{p}^{3}} \leq\left\|\mathcal{M}^{\frac{3}{2}} w\right\|_{\mathbf{L}_{p}^{2}} .
$$

Taking $\alpha_{2}=\min \left(\alpha_{1}, a_{2}, a_{3}\right)$, we conclude the proof of Proposition 3.1. 


\subsection{Proof of Theorem 2}

We fix $h_{a}^{\max }$, with $0<h_{a}^{\max }<\frac{\alpha \kappa}{2}$, and $\theta_{\max }=\frac{1}{2} \arcsin \left(\frac{2 h_{a}^{\max }}{\alpha \kappa}\right)$. We introduce the constants $\alpha_{1}$, $\alpha_{2}$, and $\alpha_{3}$ given by Proposition 3.1.

We consider $v$ a solution of (3.15). We define $\sigma(t)$ by

$$
\sigma(t)=\frac{1}{4 \pi} \int_{\Omega} \frac{v(t, x, y)}{\cosh x} d x d y
$$

so that we can split $v$ as

$$
v(t, x, y)=w(t, x, y)+\frac{\sigma(t)}{\cosh x}\left(\begin{array}{l}
1 \\
0
\end{array}\right), \text { where } w(t, \cdot) \in H_{p}^{1, \perp} .
$$

We plug (3.26) in (3.15). Since $\mathcal{M}\left(\begin{array}{c}\frac{1}{\cosh x} \\ 0\end{array}\right)=\ell\left(\begin{array}{c}\frac{1}{\cosh x} \\ 0\end{array}\right)=0$, we have:

$$
\frac{\partial w}{\partial t}+\frac{1}{\cosh x} \frac{d \sigma}{d t}\left(\begin{array}{l}
1 \\
0
\end{array}\right)=\left(\begin{array}{cc}
-\alpha & -1 \\
1 & -\alpha
\end{array}\right) \mathcal{M} w-h_{a} \mathcal{L} w
$$

We take the $L^{2}$-inner product of $(3.27)$ with $\frac{1}{4 \pi \cosh x}\left(\begin{array}{l}1 \\ 0\end{array}\right)$. We remark that

- $\left\langle w_{1} \mid \frac{1}{\cosh x}\right\rangle=0$ for all $t$, so $\left\langle\partial_{t} w_{1} \mid \frac{1}{\cosh x}\right\rangle=0$,

- $\left\langle\partial_{y y} w_{i} \mid \frac{1}{\cosh x}\right\rangle=\left\langle\tanh x \partial_{y} w_{i} \mid \frac{1}{\cosh x}\right\rangle=0$, by integration of parts in $y$ and $2 \pi$-periodicity,

- $L$ is self-adjoint so that $\left\langle L w_{i} \mid \frac{1}{\cosh x}\right\rangle=\left\langle w_{i} \mid L\left(\frac{1}{\cosh x}\right)\right\rangle=0$,

- $\left\langle\frac{1}{\cosh x} \mid \frac{1}{4 \pi \cosh x}\right\rangle=1$.

Therefore we obtain:

$$
\frac{d \sigma}{d t}=\mathcal{K} w
$$

with $\mathcal{K} w=\left\langle\frac{1}{4 \pi \cosh x} \mid-\kappa \cos 2 \theta w_{2}-h_{a}\left(\alpha+\frac{1}{\alpha}\right) \ell w_{1}+2 h_{a} \tanh x w_{2}\right\rangle$. By subtraction, we obtain that

$$
\frac{\partial w}{\partial t}=\left(\begin{array}{cc}
-\alpha & -1 \\
1 & -\alpha
\end{array}\right) \mathcal{M} w-h_{a} \mathcal{L} w-\frac{1}{\cosh x}\left(\begin{array}{l}
1 \\
0
\end{array}\right) \mathcal{K} w
$$

We take the inner product of (3.29) with $\mathcal{M} w$. Since $L$ is self adjoint, since $L\left(\frac{1}{\cosh x}\right)=0$, and by integration by parts in the $y$ variable, we remark that

$$
\left\langle\frac{1}{\cosh x} \mid(\mathcal{M} w)_{1}\right\rangle=\left\langle\frac{1}{\cosh x} \mid \frac{1}{\delta^{2}} L w_{1}-\partial_{y y} w_{1}-2 \tanh x \partial_{y} w_{2}\right\rangle=0 .
$$

Therefore, we obtain that

$$
\frac{1}{2} \frac{d}{d t}\left\|\mathcal{M}^{\frac{1}{2}} w\right\|_{\mathbf{L}_{p}^{2}}^{2}+\alpha\|\mathcal{M} w\|_{\mathbf{L}_{p}^{2}}^{2}=-h_{a}\langle\mathcal{L} w \mid \mathcal{M} w\rangle
$$

Since $\mathcal{L}$ is an order one operator, there exists $K$ such that:

$$
\|\mathcal{L} w\|_{\mathbf{L}_{p}^{2}} \leq K\|w\|_{\mathbf{H}_{p}^{1}} \leq K\|w\|_{\mathbf{H}_{p}^{2}} .
$$


The equivalence of norms in Proposition 3.1 yields:

$$
\frac{1}{2} \frac{d}{d t}\left\|\mathcal{M}^{\frac{1}{2}} w\right\|_{\mathbf{L}_{p}^{2}}^{2}+\alpha\|\mathcal{M} w\|_{\mathbf{L}_{p}^{2}}^{2} \leq\left|h_{a}\right| \frac{K}{\alpha_{2}}\|\mathcal{M} w\|_{\mathbf{L}_{p}^{2}}^{2}
$$

We set $h_{\max }^{l}=\inf \left\{h_{a}^{\max }, \frac{\alpha \alpha_{2}}{2 K}\right\}$, and we get that if $\left|h_{a}\right| \leq h_{\max }^{l}$, then for all $t$,

$$
\frac{1}{2} \frac{d}{d t}\left\|\mathcal{M}^{\frac{1}{2}} w\right\|_{\mathbf{L}_{p}^{2}}^{2}+\frac{\alpha}{2}\|\mathcal{M} w\|_{\mathbf{L}_{p}^{2}}^{2} \leq 0
$$

Using again Proposition 3.1, we obtain that

$$
\frac{d}{d t}\left\|\mathcal{M}^{\frac{1}{2}} w\right\|_{\mathbf{L}_{p}^{2}}^{2}+\alpha\left(\alpha_{1}\right)^{2}\left\|\mathcal{M}^{\frac{1}{2}} w\right\|_{\mathbf{L}_{p}^{2}}^{2} \leq 0,
$$

so

$$
\forall t \geq 0, \quad\left\|\mathcal{M}^{\frac{1}{2}} w\right\|_{\mathbf{L}_{p}^{2}} \leq\left\|\mathcal{M}^{\frac{1}{2}} w(0)\right\|_{\mathbf{L}_{p}^{2}} e^{-\frac{\alpha\left(\alpha_{1}\right)^{2}}{2} t}
$$

This implies that:

$$
\forall t \geq 0, \quad\|w(t)\|_{\mathbf{H}_{p}^{1}} \leq \frac{\alpha_{3}}{\alpha_{2}}\|w(0)\|_{\mathbf{H}_{p}^{1}} e^{-\frac{\alpha\left(\alpha_{1}\right)^{2}}{2} t}
$$

In addition, using that $|\mathcal{K} w(t)| \leq K\|w(t)\|_{\mathbf{H}_{p}^{1}}$ and Equation (3.28), we obtain that $\frac{d \sigma}{d t}$ is integrable on $\mathbb{R}^{+}$, so $\sigma(t)$ tends to a limit $\sigma_{\infty}$ when $t$ tends to $+\infty$. This concludes the proof of Theorem 2 .

\section{Proof of the nonlinear stability}

\subsection{New unknowns}

We remark that our model (2.11) is invariant by translation in the $x$-variable so that $x \mapsto \mathbf{M}_{0}(x-\sigma)$ is a static solution for $(2.11)$ for all $\sigma \in \mathbb{R}$. By projection on the mobile frame $\left(\mathbf{M}_{1}, \mathbf{M}_{2}\right)$, this induces the existence of a one-parameter family of static solutions for (2.12) given by

$$
R(\sigma)(x)=\left(\begin{array}{c}
\rho(\sigma)(x) \\
0
\end{array}\right)
$$

where $\rho(s)(x)=\left(M_{0}(x-\sigma) \cdot M_{1}(x)\right)=-\frac{\tanh (x-s)}{\cosh x}+\frac{\tanh x}{\cosh (x-s)}$.

The existence of this one-parameter family of solutions induces that 0 is in the spectrum of the operator arising in the linearization of (2.12), as observed in Section 3 (see also [4, 10, 11]).

Remark 4.1. In [9, 10, 11, 12], in the case of wires with circular cross section, the model is also invariant by rotation around the wire axis, so that 0 is an eigenvalue of multiplicity two of the linearization

In order to take into account this null eigenvalue, we rewrite $r$ in the following new system of coordinates:

$$
r(t, x, y)=R(\sigma(t))(x)+w(t, x, y),
$$

where for all $t, w(t, \cdot) \in \mathcal{H}_{p}^{2, \perp}$, i.e. its first component $w_{1}$ satisfies:

$$
\forall t \geq 0, \quad \int_{\Omega} w_{1}(t, x, y) \frac{1}{\cosh x} \mathrm{~d} x \mathrm{~d} y=0 .
$$

The validity of this system of coordinates is claimed in the following: 
Proposition 4.1. There exists $\xi_{0}>0$ such that for all $r \in \mathbf{L}_{p}^{2}$ with $\|r\|_{\mathbf{L}_{p}^{2}} \leq \xi_{0}$, then there exists one and only one couple $(\sigma, w) \in \mathbb{R} \times \mathbf{L}_{p}^{2, \perp}$ such that

$$
r(x, y)=R(\sigma)(x)+w(x, y) .
$$

In addition, if $r \in \mathbf{H}_{p}^{k}$ then $w \in \mathbf{H}_{p}^{k, \perp}$.

Proof. Proceeding as in [4] we define $\psi: \mathbb{R} \rightarrow \mathbb{R}$ by

$$
\psi(s)=\int_{\mathbb{R} \times] 0,2 \pi[} \rho(s)(x) \frac{1}{\cosh x} \mathrm{~d} x=2 \pi \int_{x \in \mathbb{R}}\left(-\frac{\tanh (x-s)}{\cosh ^{2} x}+\frac{\tanh x}{\cosh (x-s) \cosh x}\right) \mathrm{d} x .
$$

We remark that if $r$ admits a decomposition on the form $r(x, y)=R(\sigma)(x)+w(x, y)$ with $w \in \mathbf{L}_{p}^{2, \perp}$ then

$$
\int_{\mathbb{R} \times] 0,2 \pi[} r_{1}(x, y) \frac{1}{\cosh x} \mathrm{~d} x \mathrm{~d} y=\psi(\sigma) .
$$

Since $\psi(0)=0$ and $\psi^{\prime}(0)=4 \pi \neq 0, \psi$ is a $\mathcal{C}^{\infty}$-diffeomorphism in a neighborhood of zero, so that for $r$ small enough, $\sigma$ is characterized by:

$$
\sigma=\psi^{-1}\left(\int_{\mathbb{R} \times] 0,2 \pi[} r_{1}(x, y) \frac{1}{\cosh x} \mathrm{~d} x \mathrm{~d} y\right) .
$$

By subtraction we obtain then that $w$ is characterized by $w=r-R(\sigma)$ which is automatically in $\mathbf{L}_{p}^{2, \perp}$.

The $\mathbf{H}_{p}^{k}$-regularity is a straightforward consequence of the previous decomposition since $R(\sigma)$ is smooth.

We aim to establish the equivalent formulation for Equation (2.12) in the new variables $(\sigma, w)$. For a fixed $\bar{\sigma} \in \mathbb{R}, R(\bar{\sigma})$ is a static solution for (2.12), so for all $t$ we have

$$
\left(\begin{array}{cc}
-\alpha & -1 \\
1 & -\alpha
\end{array}\right) \mathcal{M} R(\sigma(t))-h_{a} \mathcal{L} R(\sigma(t))+\mathcal{F}(R(\sigma(t)))=0 .
$$

Therefore plugging (4.32) in (2.11) we obtain

$$
\partial_{s} R(\sigma) \frac{d \sigma}{d t}+\partial_{t} w=\left(\begin{array}{cc}
-1 & -1 \\
1 & -1
\end{array}\right) \mathcal{M} w-h_{a} \mathcal{L} w+\mathcal{G},
$$

where $\mathcal{G}=\mathcal{F}(R(\sigma)+w)-\mathcal{F}(R(\sigma))$. We take the $\mathbf{L}_{p}^{2}$-inner product of (4.34) with $\frac{1}{4 \pi \cosh x}\left(\begin{array}{l}1 \\ 0\end{array}\right)$, and using the same arguments as in Section 3.2, we obtain:

$$
g(\sigma) \frac{d \sigma}{d t}=-h_{a} \mathcal{K} w+\tilde{G}
$$

where

- $g(s)=\int_{x \in \mathbb{R}} \frac{1}{2 \cosh x} \partial_{s} \rho(s)(x) \mathrm{d} x=1+\mathcal{O}(s)$, so that $g(s) \geq \frac{1}{2}$ for $\sigma$ small enough,

- $\mathcal{K} w=\int_{\Omega} \frac{1}{4 \pi \cosh x}\left(-\kappa \cos 2 \theta w_{2}-h_{a}\left(\alpha+\frac{1}{\alpha}\right) \ell w_{1}+2 h_{a} \tanh x w_{2}\right) \mathrm{d} x \mathrm{~d} y$,

- $\tilde{G}=\int_{\Omega} \frac{1}{4 \pi \cosh x} \mathcal{G}_{1} \mathrm{~d} x \mathrm{~d} y$ where $\mathcal{G}_{1}$ is the first component of $\mathcal{G}$. 
Therefore we get

$$
\frac{d \sigma}{d t}=\mathcal{K}_{\sigma} w+G
$$

where $\mathcal{K}_{\sigma} w=\frac{\mathcal{K} w}{g(\sigma)}$ and $G=\frac{\tilde{G}}{g(\sigma)}$.

Writing in (4.34) that $\partial_{s} \rho(\sigma)=\frac{1}{\cosh x}+a(x, \sigma)$ where $a(x, \sigma)=\mathcal{O}(\sigma)$, using $(4.35)$, we obtain by subtraction that :

$$
\partial_{t} w=\left(\begin{array}{cc}
-1 & -1 \\
1 & -1
\end{array}\right) \mathcal{M} w-h_{a} \mathcal{L} w-\frac{1}{\cosh x}\left(\begin{array}{l}
1 \\
0
\end{array}\right) \mathcal{K}_{\sigma} w+\mathcal{H}
$$

where

$$
\mathcal{H}=\mathcal{G}-a(x, \sigma)\left(\begin{array}{c}
\mathcal{K}_{\sigma} w \\
0
\end{array}\right)-\left(\begin{array}{c}
\partial_{s} \rho(\sigma) G \\
0
\end{array}\right) .
$$

In order to avoid the singularity of $\mu$, we have to assume that $\|r\|_{L^{\infty}} \leq \frac{1}{2}$. In addition, we must assume that $\|r\|_{\mathbf{L}_{p}^{2}} \leq \xi_{0}$ to use Proposition 4.1, and that $|\sigma|$ small enough to be sure that $g(\sigma) \geq \frac{1}{2}$. Therefore, using the Sobolev embedding of $\mathbf{H}_{p}^{2}$ into $L^{\infty}\left(\mathbb{R}^{2}\right)$, we introduce $\eta_{0}>0$ such that under the assumption:

$$
|\sigma| \leq \eta_{0} \quad \text { and } \quad\|w\|_{\mathbf{H}_{p}^{2}} \leq \eta_{0}
$$

then we have:

$$
\|R(\sigma)+w\|_{L^{\infty}} \leq \frac{1}{2}, \quad\|R(\sigma)+w\|_{\mathbf{L}_{p}^{2}} \leq \frac{1}{2}, \quad \text { and } \quad g(\sigma) \geq \frac{1}{2}
$$

\subsection{Nonlinear Stability}

We fix an a priori bound on $h_{a}$ : let $h_{a}^{\max }$ satisfying $0<h_{a}^{\max }<\frac{\alpha \kappa}{2}$ and let $\theta_{\max }$ related to $h_{a}^{\max }$ by $(1.10)$ :

$$
\theta_{\max }=\frac{1}{2} \arcsin \left(\frac{2 h_{a}^{\max }}{\kappa}\right) .
$$

We introduce the constants $\alpha_{1}, \alpha_{2}$ and $\alpha_{3}$ given by Proposition 3.1 with this $\theta_{\max }$, so that the norms equivalences in Proposition 3.1 are valid for all $\theta$ with $|\theta| \leq \theta_{\max }$, i.e. for all $h_{a}$ with $\left|h_{a}\right| \leq h_{a}^{\max }$.

We aim to perform variational estimates on System (4.35)-(4.36). The right-hand side nonlinear terms are estimated in the following Proposition:

Proposition 4.2. There exists a constant $K$ such that for all $h_{a}$ satisfying $\left|h_{a}\right| \leq h_{a}^{\max }$, for all $\sigma \in \mathbb{R}$ satisfying $|\sigma| \leq \eta_{0}$, for all $w \in \mathbf{H}_{p}^{3}$ such that $\|w\|_{\mathbf{H}_{p}^{2}} \leq \eta_{0}$, then

$$
\begin{gathered}
\left|\mathcal{K}_{\sigma}(w)\right| \leq K\|w\|_{\mathbf{H}_{p}^{2}}, \quad|G| \leq K\|w\|_{\mathbf{H}_{p}^{2}}, \quad\|\mathcal{H}\|_{\mathbf{L}_{p}^{2}} \leq K\left(|\sigma|+\|w\|_{\mathbf{H}_{p}^{2}}\right)\|w\|_{\mathbf{H}_{p}^{2}}, \\
\text { and } \quad\|\mathcal{H}\|_{\mathbf{H}_{p}^{1}} \leq K\left(|\sigma|+\|w\|_{\mathbf{H}_{p}^{2}}\right)\|w\|_{\mathbf{H}_{p}^{3}} .
\end{gathered}
$$

For the convenience of the reader the proof of this proposition is postponed to Section 5 .

First step: $H^{1}$ estimates. Taking the inner product of (4.36) with $\mathcal{M} w$, since the first component of $\mathcal{M} w$ is orthogonal to $\frac{1}{\cosh x}$, we obtain:

$$
\frac{1}{2} \frac{d}{d t}\langle w \mid \mathcal{M} w\rangle+\alpha\|\mathcal{M} w\|_{\mathbf{L}_{p}^{2}}^{2}=-h_{a}\langle\mathcal{L} w \mid \mathcal{M} w\rangle+\langle\mathcal{H} \mid \mathcal{M} w\rangle .
$$

By Proposition 4.2 and by the norms equivalences established in Proposition 3.1, we obtain that there exists $K_{1}$ such that while $|\sigma| \leq \eta_{0}$ and $\|w\|_{\mathbf{H}_{p}^{2}} \leq \eta_{0}$, then

$$
\|\mathcal{L} w\|_{\mathbf{L}_{p}^{2}} \leq K_{1}\|\mathcal{M} w\|_{\mathbf{L}_{p}^{2}} \quad \text { and } \quad\|\mathcal{H}\|_{\mathbf{L}_{p}^{2}} \leq K_{1}\left(|\sigma|+\|\mathcal{M} w\|_{\mathbf{L}_{p}^{2}}\right)\|\mathcal{M} w\|_{\mathbf{L}_{p}^{2}}
$$


So

$$
\frac{1}{2} \frac{d}{d t}\langle w \mid \mathcal{M} w\rangle+\alpha\|\mathcal{M} w\|_{\mathbf{L}_{p}^{2}}^{2} \leq K_{1}\left(h_{a}+|\sigma|+\|\mathcal{M} w\|_{\mathbf{L}_{p}^{2}}\right)\|\mathcal{M} w\|_{\mathbf{L}_{p}^{2}}^{2}
$$

Second step: $H^{2}$ estimates. We will take the inner product of (4.36) with $\mathcal{M}^{2} w$. Denoting by $Y_{1}$ and $Y_{2}$ the coordinates of $\mathcal{M} w$, we have on the one hand:

$$
\begin{aligned}
\left\langle\left(\begin{array}{cc}
-\alpha & -1 \\
1 & -\alpha
\end{array}\right) \mathcal{M} w \mid \mathcal{M}^{2} w\right\rangle & =\left\langle\left(\begin{array}{c}
-\alpha Y_{1}-Y_{2} \\
Y_{1}-\alpha Y_{2}
\end{array}\right) \mid \mathcal{M} Y\right\rangle \\
& =-\alpha\langle Y \mid \mathcal{M} Y\rangle-\left\langle Y_{2} \mid(\mathcal{M} Y)_{1}\right\rangle+\left\langle Y_{1} \mid(\mathcal{M} Y)_{2}\right\rangle .
\end{aligned}
$$

Now,

$$
\begin{aligned}
-\left\langle Y_{2} \mid(\mathcal{M} Y)_{1}\right\rangle & =-\left\langle Y_{2} \mid\left(\frac{1}{\delta^{2}} L-\partial_{y y}\right) Y_{1}+2 \tanh x \partial_{y} Y_{2}\right\rangle, \\
& =-\left\langle\left(\frac{1}{\delta^{2}} L-\partial_{y y}\right) Y_{2} \mid Y_{1}\right\rangle,
\end{aligned}
$$

since $\frac{1}{\delta^{2}} L-\partial_{y y}$ is self-adjoint and by $2 \pi$-periodicity of $Y_{2}$, so that $\left\langle Y_{2} \mid \tanh x \partial_{y} Y_{2}\right\rangle=0$.

In addition, with the same arguments,

$$
\begin{aligned}
\left\langle Y_{1} \mid(\mathcal{M} Y)_{2}\right\rangle & =\left\langle Y_{1} \mid\left(\frac{1}{\delta^{2}} L-\partial_{y y}\right) Y_{2}+\kappa \cos 2 \theta Y_{2}-2 \tanh x \partial_{y} Y_{1}\right\rangle \\
& =\left\langle Y_{1} \mid\left(\frac{1}{\delta^{2}} L-\partial_{y y}\right) Y_{2}\right\rangle+\kappa \cos 2 \theta\left\langle Y_{1} \mid Y_{2}\right\rangle .
\end{aligned}
$$

Hence

$$
\left\langle\left(\begin{array}{cc}
-\alpha & -1 \\
1 & -\alpha
\end{array}\right) \mathcal{M} w \mid \mathcal{M}^{2} w\right\rangle=-\alpha\left\|\mathcal{M}^{\frac{1}{2}} Y\right\|_{\mathbf{L}_{p}^{2}}^{2}+\kappa \cos 2 \theta\left\langle Y_{1} \mid Y_{2}\right\rangle
$$

On the other hand,

$$
\left\langle\frac{1}{\cosh x} \mid(\mathcal{M} Y)_{1}\right\rangle=\left\langle\frac{1}{\cosh x} \mid \frac{1}{\delta^{2}} L Y_{1}-\partial_{y y} Y_{1}+2 \tanh x \partial_{y} Y_{2}\right\rangle=0
$$

Therefore by taking the inner product of (4.36) with $\mathcal{M}^{2} w$, we obtain:

$$
\begin{aligned}
\frac{1}{2} \frac{d}{d t}\|\mathcal{M} w\|_{\mathbf{L}_{p}^{2}}^{2}+\alpha\left\|\mathcal{M}^{\frac{3}{2}} w\right\|_{\mathbf{L}_{p}^{2}}^{2} & =\kappa \cos 2 \theta\left\langle(\mathcal{M} w)_{1} \mid(\mathcal{M} w)_{2}\right\rangle-h_{a}\left\langle\mathcal{L} w \mid \mathcal{M}^{2} w\right\rangle+\left\langle\mathcal{H} \mid \mathcal{M}^{2} w\right\rangle \\
& \leq \kappa\|\mathcal{M} w\|_{\mathbf{L}_{p}^{2}}^{2}-h_{a}\left\langle\mathcal{M}^{\frac{1}{2}} \mathcal{L} w \mid \mathcal{M}^{\frac{3}{2}} w\right\rangle+\left\langle\mathcal{M}^{\frac{1}{2}} \mathcal{H} \mid \mathcal{M}^{\frac{3}{2}} w\right\rangle
\end{aligned}
$$

Using Propositions 3.1 and 4.2, while $|\sigma| \leq \eta_{0}$ and $\|w\|_{\mathbf{H}_{p}^{2}} \leq \eta_{0}$, there exists $K_{2}$ such that

$$
\left\|\mathcal{M}^{\frac{1}{2}} \mathcal{L} w\right\|_{\mathbf{L}_{p}^{2}} \leq K_{2}\left\|\mathcal{M}^{\frac{3}{2}} w\right\|_{\mathbf{L}_{p}^{2}}
$$

and

$$
\left\|\mathcal{M}^{\frac{1}{2}} \mathcal{H}\right\|_{\mathbf{L}_{p}^{2}} \leq K_{2}\left(|\sigma|+\|\mathcal{M} w\|_{\mathbf{L}_{p}^{2}}\right)\left\|\mathcal{M}^{\frac{3}{2}} w\right\|_{\mathbf{L}_{p}^{2}}
$$

Therefore we obtain that while $|\sigma| \leq \eta_{0}$ and $\|w\|_{\mathbf{H}_{p}^{2}} \leq \eta_{0}$,

$$
\frac{1}{2} \frac{d}{d t}\|\mathcal{M} w\|_{\mathbf{L}_{p}^{2}}^{2}+\alpha\left\|\mathcal{M}^{\frac{3}{2}} w\right\|_{\mathbf{L}_{p}^{2}}^{2} \leq \kappa\|\mathcal{M} w\|_{\mathbf{L}_{p}^{2}}^{2}+K_{2}\left(h_{a}+|\sigma|+\|\mathcal{M} w\|_{\mathbf{L}_{p}^{2}}\right)\left\|\mathcal{M}^{\frac{3}{2}} w\right\|_{\mathbf{L}_{p}^{2}}^{2} .
$$


Adding up $2 \kappa \times(4.40)$ and $\alpha \times(4.41)$ (so that the right-hand side term $\kappa\|\mathcal{M} w\|_{\mathbf{L}_{p}^{2}}^{2}$ in (4.41) is absorbed by the left-hand side of (4.40)), we obtain that while $|\sigma| \leq \eta_{0}$ and $\|w\|_{\mathbf{H}_{p}^{2}} \leq \eta_{0}$,

$$
\begin{aligned}
& \frac{1}{2} \frac{d}{d t}\left(2 \kappa\langle w \mid \mathcal{M} w\rangle+\alpha\|\mathcal{M} w\|_{\mathbf{L}_{p}^{2}}^{2}\right)+\alpha \kappa\|\mathcal{M} w\|_{\mathbf{L}_{p}^{2}}^{2}+\alpha^{2}\left\|\mathcal{M}^{\frac{3}{2}} w\right\|_{\mathbf{L}_{p}^{2}}^{2} \\
& \quad \leq 2 \kappa K_{1}\left(h_{a}+|\sigma|+\|\mathcal{M} w\|_{\mathbf{L}_{p}^{2}}\right)\|\mathcal{M} w\|_{\mathbf{L}_{p}^{2}}^{2}+\alpha K_{2}\left(h_{a}+|\sigma|+\|\mathcal{M} w\|_{\mathbf{L}_{p}^{2}}\right)\left\|\mathcal{M}^{\frac{3}{2}} w\right\|_{\mathbf{L}_{p}^{2}}^{2},
\end{aligned}
$$

that is

$$
\begin{array}{r}
\frac{1}{2} \frac{d}{d t}\left(2 \kappa\langle w \mid \mathcal{M} w\rangle+\alpha\|\mathcal{M} w\|_{\mathbf{L}_{p}^{2}}^{2}\right)+\kappa\left(\alpha-2 K_{1} h_{a}-2 K_{1}|\sigma|-2 K_{1}\|\mathcal{M} w\|_{\mathbf{L}_{p}^{2}}\right)\|\mathcal{M} w\|_{\mathbf{L}_{p}^{2}}^{2} \\
+\alpha\left(\alpha-K_{2} h_{a}-K_{2}|\sigma|-K_{2}\|\mathcal{M} w\|_{\mathbf{L}_{p}^{2}}\right)\left\|\mathcal{M}^{\frac{3}{2}} w\right\|_{\mathbf{L}_{p}^{2}}^{2} \leq 0 .
\end{array}
$$

We fix $h_{\max }$ by:

$$
h_{\max }=\min \left\{h_{a}^{\max }, \frac{\alpha}{4 K_{1}}, \frac{\alpha}{2 K_{2}}\right\} .
$$

From now on, we assume that

$$
\left|h_{a}\right| \leq h_{\max }
$$

We obtain that while $|\sigma| \leq \eta_{0}$ and $\|w\|_{\mathbf{H}_{p}^{2}} \leq \eta_{0}$ :

$$
\begin{array}{r}
\frac{1}{2} \frac{d}{d t}\left(2 \kappa\langle w \mid \mathcal{M} w\rangle+\alpha\|\mathcal{M} w\|_{\mathbf{L}_{p}^{2}}^{2}\right)+\kappa\left(\frac{\alpha}{2}-2 K_{1}|\sigma|-2 K_{1}\|\mathcal{M} w\|_{\mathbf{L}_{p}^{2}}\right)\|\mathcal{M} w\|_{\mathbf{L}_{p}^{2}}^{2} \\
+\alpha\left(\frac{\alpha}{2}-K_{2}|\sigma|-K_{2}\|\mathcal{M} w\|_{\mathbf{L}_{p}^{2}}\right)\left\|\mathcal{M}^{\frac{3}{2}} w\right\|_{\mathbf{L}_{p}^{2}}^{2} \leq 0 .
\end{array}
$$

Third step: joint estimates for $w$ and $\sigma$. We fix $\eta_{1}=\min \left\{\eta_{0}, \frac{\alpha}{16 K_{1}}, \frac{\alpha}{8 K_{2}}\right\}$. While $|\sigma| \leq \eta_{1}$ and $\|\mathcal{M} w\|_{\mathbf{L}_{p}^{2}} \leq \eta_{1}$ then:

$$
\frac{1}{2} \frac{d}{d t}\left(2 \kappa\langle w \mid \mathcal{M} w\rangle+\alpha\|\mathcal{M} w\|_{\mathbf{L}_{p}^{2}}^{2}\right)+\frac{\alpha \kappa}{4}\|\mathcal{M} w\|_{\mathbf{L}_{p}^{2}}^{2}+\frac{\alpha^{2}}{4}\left\|\mathcal{M}^{\frac{3}{2}} w\right\|_{\mathbf{L}_{p}^{2}}^{2} \leq 0 .
$$

Therefore, using Proposition 3.1, we obtain that while $|\sigma| \leq \eta_{1}$ and $\|\mathcal{M} w\|_{\mathbf{L}_{p}^{2}} \leq \eta_{1}$ then:

$$
\frac{1}{2} \frac{d}{d t}\left(2 \kappa\left\|\mathcal{M}^{\frac{1}{2}} w\right\|_{\mathbf{L}_{p}^{2}}^{2}+\alpha\|\mathcal{M} w\|_{\mathbf{L}_{p}^{2}}^{2}\right)+\frac{\alpha\left(\alpha_{1}\right)^{2}}{8}\left(2 \kappa\left\|\mathcal{M}^{\frac{1}{2}} w\right\|_{\mathbf{L}_{p}^{2}}^{2}+\alpha\|\mathcal{M} w\|_{\mathbf{L}_{p}^{2}}^{2}\right) \leq 0,
$$

so that, by comparison lemma, while $|\sigma| \leq \eta_{1}$ and $\|\mathcal{M} w\|_{\mathbf{L}_{p}^{2}} \leq \eta_{1}$ then:

$$
\left(2 \kappa\left\|\mathcal{M}^{\frac{1}{2}} w(t)\right\|_{\mathbf{L}_{p}^{2}}^{2}+\alpha\|\mathcal{M} w(t)\|_{\mathbf{L}_{p}^{2}}^{2}\right) \leq\left(2 \kappa\left\|\mathcal{M}^{\frac{1}{2}} w(0)\right\|_{\mathbf{L}_{p}^{2}}^{2}+\alpha\|\mathcal{M} w(0)\|_{\mathbf{L}_{p}^{2}}^{2}\right) e^{-\frac{\alpha\left(\alpha_{1}\right)^{2}}{4} t} .
$$

From (4.35) we have:

$$
\left|\frac{d \sigma}{d t}\right| \leq\left|\mathcal{K}_{\sigma} w\right|+|\mathcal{G}|,
$$

hence, using Propositions 3.1 and 4.2, there exists a constant $K_{3}$ such that:

$$
\left|\frac{d \sigma}{d t}\right| \leq K_{3}\|\mathcal{M} w\|_{\mathbf{L}_{p}^{2}} .
$$

Therefore, while $|\sigma| \leq \eta_{1}$ and $\|\mathcal{M} w\|_{\mathbf{L}_{p}^{2}} \leq \eta_{1}$, then

$$
\left|\frac{d \sigma}{d t}\right| \leq K_{3}\left(2 \kappa\left\|\mathcal{M}^{\frac{1}{2}} w(0)\right\|_{\mathbf{L}_{p}^{2}}^{2}+\alpha\|\mathcal{M} w(0)\|_{\mathbf{L}_{p}^{2}}^{2}\right)^{\frac{1}{2}} \exp \left(-\frac{\alpha\left(\alpha_{1}\right)^{2}}{8} t\right),
$$


so, by integration,

$$
|\sigma(t)| \leq|\sigma(0)|+\frac{8 K_{3}}{\alpha\left(\alpha_{1}\right)^{2}}\left(2 \kappa\left\|\mathcal{M}^{\frac{1}{2}} w(0)\right\|_{\mathbf{L}_{p}^{2}}^{2}+\alpha\|\mathcal{M} w(0)\|_{\mathbf{L}_{p}^{2}}^{2}\right)^{\frac{1}{2}} .
$$

End of the proof. Using Proposition 3.1, we introduce $\eta_{2}>0$ such that for any $w$, if $\|w(0)\|_{\mathbf{H}_{p}^{2}} \leq$ $\eta_{2}$, then

$$
\left(1+\frac{8 K_{3}}{\alpha\left(\alpha_{1}\right)^{2}}\right)\left(2 \kappa\left\|\mathcal{M}^{\frac{1}{2}} w(0)\right\|_{\mathbf{L}_{p}^{2}}^{2}+\alpha\|\mathcal{M} w(0)\|_{\mathbf{L}_{p}^{2}}^{2}\right)^{\frac{1}{2}} \leq \frac{\eta_{1}}{4} .
$$

We assume that $|\sigma(0)| \leq \frac{\eta_{1}}{4}$ and that $\|w(0)\|_{\mathbf{H}_{p}^{2}} \leq \eta_{2}$. Let us prove that for all $t \geq 0$, we have: $|\sigma(t)|<\eta$ and $\|\mathcal{M}(w(t))\|_{\mathbf{L}_{p}^{2}}<\eta_{1}$.

If not, since this property is obviously satisfied at $t=0$, we introduce $t_{1}>0$ the first time in which this property fails. In particular, we have either $\left|\sigma\left(t_{1}\right)\right|=\eta_{1}$ or $\left\|\mathcal{M}\left(w\left(t_{1}\right)\right)\right\|_{\mathbf{L}_{p}^{2}}=\eta_{1}$.

For all $t<t_{1}$, we have $|\sigma(t)| \leq \eta_{1}$ and $\|\mathcal{M} w(t)\|_{\mathbf{L}_{p}^{2}} \leq \eta_{1}$, so that Estimates (4.42) and (4.45) are valid on this interval. In particular, at $t=t_{1}$, by continuity we have:

$$
\left\|\mathcal{M} w\left(t_{1}\right)\right\|_{\mathbf{L}_{p}^{2}} \leq\left(2 \kappa\left\|\mathcal{M}^{\frac{1}{2}} w(0)\right\|_{\mathbf{L}_{p}^{2}}^{2}+\alpha\|\mathcal{M} w(0)\|_{\mathbf{L}_{p}^{2}}^{2}\right)^{\frac{1}{2}} \leq \frac{\eta_{1}}{4}
$$

and

$$
\left|\sigma\left(t_{1}\right)\right| \leq|\sigma(0)|+\frac{\eta_{1}}{4} \leq \frac{\eta_{1}}{2}
$$

and this leads to a contradiction.

Therefore, if $\sigma(0) \leq \frac{\eta_{1}}{4}$ and $\|w(0)\|_{\mathbf{H}_{p}^{2}} \leq \eta_{2}$, then for all $t \geq 0,(4.42)$ and (4.43) remain valid so that

- $\|w\|_{\mathbf{H}_{p}^{2}}$ remains small for all times,

- $w$ tends to zero in $\mathbf{H}_{p}^{2}$ when $t$ tends to $+\infty$,

- $\sigma$ remains small for all times,

- since $\frac{d \sigma}{d t}$ is integrable on $\mathbb{R}^{+}$by $(4.44), \sigma$ tends to a limit $\sigma_{\infty}$ when $t$ tends to $+\infty$.

This concludes the proof of Theorem 1 .

\section{Estimate of the nonlinear terms}

The aim of this part is to estimate the right-hand side terms of (4.35) and to obtain $L^{2}$ and $H^{1}$ estimates for the nonlinear terms in (4.36).

First we give the exact expression of $\mathcal{F}(r)$, the nonlinear term arising in Equation (2.12):

$$
\begin{aligned}
\mathcal{F}= & F_{1}(r)\left(\partial_{x x} r\right)+F_{2}(r)\left(\partial_{y y} r\right)+F_{3}(r)\left(\partial_{x} r, \partial_{x} r\right)+F_{4}(r)\left(\partial_{y} r, \partial_{y} r\right)+F_{5}(x, r)\left(\partial_{x} r\right) \\
& +F_{6}(x, r)\left(\partial_{y} r\right)+F_{7}(x, r)
\end{aligned}
$$

with 
- $F_{1}(r)\left(\partial_{x x} r\right)=\frac{1}{\delta^{2}}\left(\begin{array}{cc}-\alpha r_{1}^{2} & \mu(r)-\alpha r_{1} r_{2} \\ -\mu(r)-\alpha r_{1} r_{2} & -\alpha r_{2}^{2}\end{array}\right) \partial_{x x} r-\frac{1}{\delta^{2}}\left(\begin{array}{c}r_{2}+\alpha r_{1}+\alpha \mu(r) r_{1} \\ \alpha r_{2}-r_{1}+\alpha \mu(r) r_{2}\end{array}\right) \mathrm{d} \mu(r)\left(\partial_{x x} r\right)$,

- $F_{2}(r)\left(\partial_{y y} r\right)=\left(\begin{array}{cc}-\alpha r_{1}^{2} & \mu(r)-\alpha r_{1} r_{2} \\ -\mu(r)-\alpha r_{1} r_{2} & -\alpha r_{2}^{2}\end{array}\right) \partial_{y y} r-\left(\begin{array}{c}r_{2}+\alpha r_{1}+\alpha \mu(r) r_{1} \\ \alpha r_{2}-r_{1}+\alpha \mu(r) r_{2}\end{array}\right) \mathrm{d} \mu(r)\left(\partial_{y y} r\right)$,

- $F_{3}(r)\left(\partial_{x} r, \partial_{x} r\right)=-\frac{1}{\delta^{2}}\left(\begin{array}{c}r_{2}+\alpha r_{1}+\alpha \mu(r) r_{1} \\ \alpha r_{2}-r_{1}+\alpha \mu(r) r_{2}\end{array}\right) \mathrm{d}^{2} \mu(r)\left(\partial_{x} r, \partial_{x} r\right)$,

- $F_{4}(r)\left(\partial_{y} r, \partial_{y} r\right)=-\left(\begin{array}{c}r_{2}+\alpha r_{1}+\alpha \mu(r) r_{1} \\ \alpha r_{2}-r_{1}+\alpha \mu(r) r_{2}\end{array}\right) \mathrm{d}^{2} \mu(r)\left(\partial_{y} r, \partial_{y} r\right)$,

- $F_{5}(x, r)\left(\partial_{x} r\right)=-\frac{2}{\delta^{2} \cosh x}\left(\begin{array}{c}r_{2}+\alpha r_{1}+\alpha \mu(r) r_{1} \\ \alpha r_{2}-r_{1}+\alpha \mu(r) r_{2}\end{array}\right) \partial_{x} r_{1}+\frac{2}{\delta^{2} \cosh x}\left(\begin{array}{c}-\alpha+\alpha r_{1}^{2} \\ \mu(r)+\alpha r_{1} r_{2}+1\end{array}\right) \mathrm{d} \mu(r)\left(\partial_{x} r\right)$,

- $F_{6}(x, r)\left(\partial_{y} r\right)=2 \tanh x\left(\begin{array}{cc}\mu(r)-\alpha r_{1} r_{2} & \alpha r_{1}^{2} \\ -\alpha r_{2}^{2} & \mu(r)+\alpha r_{1} r_{2}\end{array}\right) \partial_{y} r+\frac{2}{\cosh x}\left(\begin{array}{c}r_{2}+\alpha r_{1}+\alpha \mu(r) r_{1} \\ \alpha r_{2}-r_{1}+\alpha \mu(r) r_{2}\end{array}\right) \partial_{y} r_{2}$

$$
+\frac{2}{\cosh x}\left(\begin{array}{c}
1+\mu(r)-\alpha r_{1} r_{2} \\
\alpha-\alpha r_{2}^{2}
\end{array}\right) \mathrm{d} \mu(r)\left(\partial_{y} r\right)
$$

- $F_{7}(x, r)=\left(\frac{2}{\delta^{2}} \frac{\tanh x}{\cosh x} r_{1}+\frac{h_{a}}{\alpha} \frac{1}{\cosh x} r_{2}-\mu(r)\left(1-\frac{2}{\cosh ^{2} x} \frac{1}{\delta^{2}}\right)\right)\left(\begin{array}{c}r_{2}+\alpha r_{1}+\alpha \mu(r) r_{1} \\ -r_{1}+\alpha r_{2}+\alpha \mu(r) r_{2}\end{array}\right)$

$$
\begin{aligned}
& -\alpha\left(1-\frac{2}{\cosh ^{2} x} \frac{1}{\delta^{2}}+h_{a} \tanh x\right) r \mu(r)+\frac{h_{a}}{\cosh x}\left(\begin{array}{c}
\alpha \\
-1
\end{array}\right) \mu(r) \\
& +\left(h_{a} \frac{1}{\cosh x}+\kappa \sin ^{2} \theta r_{1}+\frac{h_{a}}{\alpha} \tanh x r_{2}\right)\left(\begin{array}{c}
\alpha r_{1}^{2} \\
\mu(r)+\alpha r_{1} r_{2}
\end{array}\right) \\
& +\left(\frac{h_{a}}{\alpha} \frac{1}{\cosh x}+\kappa \cos ^{2} \theta r_{2}+\frac{h_{a}}{\alpha} \tanh x r_{1}+\frac{h_{a}}{\alpha} \frac{1}{\cosh x} \mu(r)\right)\left(\begin{array}{c}
\alpha r_{1} r_{2}-\mu(r) \\
\alpha r_{2}^{2}
\end{array}\right) .
\end{aligned}
$$

The term $\mathcal{G}$ arising in Equation (4.34) is defined by $\mathcal{G}=\mathcal{F}(R(\sigma)+w)-\mathcal{F}(R(\sigma))$, so that we have:

$$
\mathcal{G}=G_{1}+\ldots+G_{7}
$$

with

$$
\begin{aligned}
G_{1}= & F_{1}(R(\sigma)+w)\left(\partial_{x x} w\right)+\widetilde{F}_{1}(R(\sigma), w)(w)\left(\partial_{x x} R(\sigma)\right), \\
G_{2}= & F_{2}(R(\sigma)+w)\left(\partial_{y y} w\right), \\
G_{3}= & F_{3}(R(\sigma)+w)\left(\partial_{x} w, \partial_{x} w\right)+2 F_{3}(R(\sigma)+w)\left(\partial_{x} R(\sigma), \partial_{x} w\right) \\
& +\widetilde{F_{3}}(R(\sigma), w)(w)\left(\partial_{x} R(\sigma), \partial_{x} R(\sigma)\right), \\
G_{4}= & F_{4}(R(\sigma)+w)\left(\partial_{y} w, \partial_{y} w\right), \\
G_{5}= & F_{5}(x, R(\sigma)+w)\left(\partial_{x} w\right)+\widetilde{F}_{5}(x, R(\sigma), w)(w)\left(\partial_{x} R(\sigma)\right), \\
G_{6}= & F_{6}(x, R(\sigma)+w)\left(\partial_{y} w\right), \\
G_{7}= & \widetilde{F}_{7}(x, R(\sigma), w)(w) .
\end{aligned}
$$


Here,

$$
\widetilde{F_{i}}(R(\sigma), w)=\int_{0}^{1} \mathrm{~d}_{r} F_{i}(R(\sigma)+s w) \mathrm{d} s \quad \text { for } i \in\{1,3\}
$$

and

$$
\widetilde{F_{i}}(x, R(\sigma), w)=\int_{0}^{1} \partial_{r} F_{i}(x, R(\sigma)+s w) \mathrm{d} s \quad \text { for } i \in\{5,7\} .
$$

Remark 5.1. These terms are obtained by the Fundamental Theorem of Calculus writing for example that

$$
F_{1}(R(\sigma)+w)=F_{1}(R(\sigma))+\int_{0}^{1} \mathrm{~d} F_{1}(R(\sigma)+s w)(w) \mathrm{d} s=F_{1}(R(\sigma))+\widetilde{F_{1}}(R(\sigma), w)(w) .
$$

On the one hand we recall that

$$
R(s)(x)=\left(\begin{array}{c}
\rho(s)(x) \\
0
\end{array}\right) \quad \text { with } \rho(s)(x)=-\frac{\tanh (x-s)}{\cosh x}+\frac{\tanh x}{\cosh (x-s)} .
$$

So by direct calculations and estimates, we obtain that there exists a constant $K$ such that if $|s| \leq 1$ then

$$
\forall x \in \mathbb{R}, \quad|R(s)(x)|+\left|\partial_{x} R(s)(x)\right|+\left|\partial_{x x} R(s)(x)\right|+\left|\partial_{x x x} R(s)(x)\right| \leq \frac{K}{\cosh x}|s| .
$$

On the other hand, since $\mu(r)=\sqrt{1-\left(r_{1}\right)^{2}-\left(r_{2}\right)^{2}}-1$, there exists a constant $K$ such that if $|r| \leq \frac{1}{2}$ then

$$
|\mu(r)| \leq K|r|^{2}, \quad|\mathrm{~d} \mu(r)| \leq K|r| \quad \text { and } \quad\left|\mathrm{d}^{2} \mu(r)\right|+\left|\mathrm{d}^{3} \mu(r)\right| \leq K .
$$

Let us now estimate each term of $G$ defined by (5.47). In what follows we recall that we assume that $|\sigma| \leq \eta_{0}$ and $\|w\|_{\mathbf{H}_{p}^{2}} \leq \eta_{0}, \eta_{0}>0$ being small enough to ensure that $\|R(\sigma)+w\|_{L^{\infty}} \leq \frac{1}{2}$.

\section{- Estimate of $G_{1}$}

We recall that

$$
G_{1}=F_{1}(R(\sigma)+w)\left(\partial_{x x} w\right)+\widetilde{F}_{1}(R(\sigma), w)(w)\left(\partial_{x x} R(\sigma)\right) .
$$

Using (5.49) and the formulation of $F_{1}$ in (5.46), there exists $K$ such that if $|r| \leq \frac{1}{2}$ then

$$
\left|F_{1}(r)\right| \leq K|r|^{2}, \quad\left|\mathrm{~d} F_{1}(r)\right| \leq K|r| \quad \text { and }\left|\mathrm{d}^{2} F_{1}(r)\right| \leq K .
$$

Since $\widetilde{F_{1}}(a, b)=\int_{0}^{1} \mathrm{~d} F_{1}(a+s b) d s$, we have also

$$
\left|\widetilde{F_{1}}(a, b)\right| \leq K(|a|+|b|) \quad \text { and }\left|\mathrm{d} \widetilde{F_{1}}(a, b)\right| \leq K .
$$

Therefore we have

$$
\begin{aligned}
\left|G_{1}\right| \leq & \left|F_{1}(R(\sigma)+w)\right|\left|\partial_{x x} w\right|+\left|\widetilde{F_{1}}(R(\sigma), w)\right||w|\left|\partial_{x x} R(\sigma)\right| \\
\leq & K|R(\sigma)+w|^{2}\left|\partial_{x x} w\right|+K(|R(\sigma)|+|w|)|w|\left|\partial_{x x} R(\sigma)\right| \\
& \operatorname{using}(5.50) \text { and (5.51), } \\
\leq & K(|\sigma|+|w|)\left(\left|\partial_{x x} w\right|+|w|\right) \quad \text { using (5.48). }
\end{aligned}
$$

Thus we get:

$$
\left\|G_{1}\right\|_{\mathbf{L}_{p}^{2}} \leq K\left(|\sigma|+\|w\|_{L^{\infty}}\right)\|w\|_{\mathbf{H}_{p}^{2}}
$$


We estimate $\nabla G_{1}$ on the following way:

$$
\begin{aligned}
\left|\nabla G_{1}\right| \leq & \left|\mathrm{d} F_{1}(R(\sigma)+w)\right|\left(\left|\partial_{x} R(\sigma)\right|+|\nabla w|\right)\left|\partial_{x x} w\right|+\left|F_{1}(R(\sigma)+w)\right|\left|\nabla \partial_{x x} w\right| \\
& +\left|\mathrm{d} \widetilde{F_{1}}(R(\sigma), w)\right|\left(\left|\partial_{x} R(\sigma)\right|+|\nabla w|\right)|w|\left|\partial_{x x} R(\sigma)\right|+\left|\widetilde{F_{1}}(R(\sigma), w)\right||\nabla w|\left|\partial_{x x} R(\sigma)\right| \\
& +\left|\widetilde{F_{1}}(R(\sigma), w)\right||w|\left|\partial_{x x x} R(\sigma)\right|,
\end{aligned}
$$

so using (5.48), (5.50) and (5.51), we obtain

$$
\begin{aligned}
\left|\nabla G_{1}\right| \leq & K(|\sigma|+|w|)(|\sigma|+|\nabla w|)\left|\partial_{x x} w\right|+K(|\sigma|+|w|)\left|\nabla \partial_{x x} w\right| \\
& +K(|\sigma|+|\nabla w|)|w||\sigma|+K(|\sigma|+|w|)|\nabla w||\sigma| .
\end{aligned}
$$

Thus,

$$
\begin{aligned}
\left\|\nabla G_{1}\right\|_{\mathbf{L}_{p}^{2}} \leq & K\left(|\sigma|+\|w\|_{L^{\infty}}\right)\left(\|w\|_{\mathbf{L}_{p}^{2}}+\|\nabla w\|_{\mathbf{L}_{p}^{2}}+\left\|\partial_{x x} w\right\|_{\mathbf{L}_{p}^{2}}+\left\|\nabla \partial_{x x} w\right\|_{\mathbf{L}_{p}^{2}}\right) \\
& +K\|\nabla w\|_{L^{4}}\left\|\partial_{x x} w\right\|_{L^{4}} .
\end{aligned}
$$

We recall that in $2 \mathrm{~d}$, we have the following interpolation-type inequality:

$$
\|v\|_{L^{4}(\mathbb{R} \times] 0,2 \pi[)} \leq C\|v\|_{L^{2}(\mathbb{R} \times] 0,2 \pi[)}^{\frac{1}{2}}\|v\|_{H^{1}(\mathbb{R} \times] 0,2 \pi[)}^{\frac{1}{2}} .
$$

Using this inequality for the last term of the previous estimate of $\left\|\nabla G_{1}\right\|_{\mathbf{L}_{p}^{2}}$, we obtain that

$$
\left\|\nabla G_{1}\right\|_{\mathbf{L}_{p}^{2}} \leq K\left(|\sigma|+\|w\|_{\mathbf{H}_{p}^{2}}\right)\|w\|_{\mathbf{H}_{p}^{3}}
$$

\section{- Estimate of $G_{2}$}

We recall that $G_{2}=F_{2}(R(\sigma)+w)\left(\partial_{y y} w\right)$, with $F_{2}=\delta^{2} F_{1}$ so with the same argument as for $G_{1}$ we obtain that there exists $K$ such that if $|\sigma| \leq \eta_{0}$ and $\|w\|_{\mathbf{H}_{p}^{2}} \leq \eta_{0}$, then:

$$
\left\|G_{2}\right\|_{\mathbf{L}_{p}^{2}} \leq K\left(|\sigma|+\|w\|_{L^{\infty}}\right)\|w\|_{\mathbf{H}_{p}^{2}}
$$

and

$$
\left\|\nabla G_{2}\right\|_{\mathbf{L}_{p}^{2}} \leq K\left(|\sigma|+\|w\|_{\mathbf{H}_{p}^{2}}\right)\|w\|_{\mathbf{H}_{p}^{3}}
$$

- Estimate of $G_{3}$

We have

$G_{3}=F_{3}(R(\sigma)+w)\left(\partial_{x} w, \partial_{x} w\right)+2 F_{3}(R(\sigma)+w)\left(\partial_{x} R(\sigma), \partial_{x} w\right)+\widetilde{F}_{3}(R(\sigma), w)(w)\left(\partial_{x} R(\sigma), \partial_{x} R(\sigma)\right)$.

Using (5.49) and the formulation of $F_{3}$ in (5.46), there exists $K$ such that if $|r| \leq \frac{1}{2}$ then

$$
\left|F_{3}(r)\right| \leq K|r| \quad \text { and }\left|\mathrm{d} F_{3}(r)\right|+\left|\mathrm{d}^{2} F_{3}\right| \leq K \text {. }
$$

Since $\widetilde{F_{3}}(a, b)=\int_{0}^{1} \mathrm{~d} F_{3}(a+s b) d s$, we have also

$$
\left|\widetilde{F_{3}}(a, b)\right|+\left|\mathrm{d} \widetilde{F_{3}}(a, b)\right| \leq K
$$

Therefore we have, for $|\sigma| \leq \eta_{0}$ and $\|w\|_{\mathbf{H}_{p}^{2}} \leq \eta_{0}$, 


$$
\begin{aligned}
\left|G_{3}\right| \leq & \left|F_{3}(R(\sigma)+w)\right|\left|\partial_{x} w\right|^{2}+2\left|F_{3}(R(\sigma)+w)\right|\left|\partial_{x} R(\sigma)\right|\left|\partial_{x} w\right| \\
& +\left|\widetilde{F}_{3}(R(\sigma), w)\right||w|\left|\partial_{x} R(\sigma)\right|^{2}, \\
\leq & K(|\sigma|+|w|)\left(\left|\partial_{x} w\right|^{2}+|\sigma|\left|\partial_{x} w\right|\right)+K|w||\sigma|^{2},
\end{aligned}
$$

with (5.53) and (5.54). So,

$$
\begin{aligned}
\left\|G_{3}\right\|_{\mathbf{L}_{p}^{2}} & \leq K\left(|\sigma|+\|w\|_{L^{\infty}}\right)\left(\left\|\partial_{x} w\right\|_{L^{4}}^{2}+|\sigma|\left\|\partial_{x} w\right\|_{\mathbf{L}_{p}^{2}}\right)+K|\sigma|^{2}\|w\|_{\mathbf{L}_{p}^{2}} \\
& \leq K\left(|\sigma|+\|w\|_{\mathbf{H}_{p}^{2}}\right)\|w\|_{\mathbf{H}_{p}^{2}} .
\end{aligned}
$$

Let us estimate now $\nabla G_{3}$ :

$$
\begin{aligned}
\left|\nabla G_{3}\right| \leq & \left|\mathrm{d} F_{3}(R(\sigma)+w)\right|\left(\left|\partial_{x} R(\sigma)\right|+|\nabla w|\right)\left|\partial_{x} w\right|^{2}+2\left|F_{3}(R(\sigma)+w)\right|\left|\nabla \partial_{x} w\right|\left|\partial_{x} w\right| \\
& +2\left|\mathrm{~d} F_{3}(R(\sigma)+w)\right|\left(\left|\partial_{x} R(\sigma)\right|+|\nabla w|\right)\left|\partial_{x} R(\sigma)\right|\left|\partial_{x} w\right| \\
& +2\left|F_{3}(R(\sigma)+w)\right|\left|\partial_{x x} R(\sigma)\right|\left|\partial_{x} w\right|+2\left|F_{3}(R(\sigma)+w)\right|\left|\partial_{x} R(\sigma)\right|\left|\nabla \partial_{x} w\right| \\
& +\left|\mathrm{d} \widetilde{F}_{3}(R(\sigma), w)\right|\left(\left|\partial_{x} R(\sigma)\right|+\nabla w \mid\right)|w|\left|\partial_{x} R(\sigma)\right|^{2}+\left|\widetilde{F}_{3}(R(\sigma), w)\right||\nabla w|\left|\partial_{x} R(\sigma)\right|^{2} \\
& +2\left|\widetilde{F}_{3}(R(\sigma), w)\right||w|\left|\partial_{x x} R(\sigma)\right|\left|\partial_{x} R(\sigma)\right|, \\
\leq & K(|\sigma|+|\nabla w|)\left|\partial_{x} w\right|^{2}+K(|\sigma|+|w|)\left|\nabla \partial_{x} w\right|\left|\partial_{x} w\right|+K(|\sigma|+|\nabla w|)|\sigma|\left|\partial_{x} w\right| \\
& +K(|\sigma|+|w|)|\sigma|\left|\partial_{x} w\right|+K(|\sigma|+|w|)|\sigma|\left|\nabla \partial_{x} w\right|+K(|\sigma|+|\nabla w|)|w||\sigma|^{2} \\
& +K|\nabla w||\sigma|^{2}+K(|\sigma|+|w|)|w||\sigma|^{2} \quad \text { using (5.53) and }(5.54) .
\end{aligned}
$$

So,

$$
\begin{aligned}
\left\|\nabla G_{3}\right\|_{\mathbf{L}_{p}^{2}} \leq & K|\sigma|\left\|\partial_{x} w\right\|_{L^{4}}^{2}+K\|\nabla w\|_{L^{6}}^{2}+K\left(|\sigma|+\|w\|_{L^{\infty}}\right)\left\|\nabla \partial_{x} w\right\|_{L^{4}}\left\|\partial_{x} w\right\|_{L^{4}} \\
& +K|\sigma|^{2}\left\|\partial_{x} w\right\|_{L^{2}}+K\|\nabla w\|_{L^{4}}^{2}|\sigma|+K\left(|\sigma|+\|w\|_{L^{\infty}}\right)|\sigma|\left\|\partial_{x} w\right\|_{L^{2}} \\
& +K\left(|\sigma|+\|w\|_{L^{\infty}}\right)|\sigma|\left\|\nabla \partial_{x} w\right\|_{L^{2}}+K|\sigma|^{3}\|w\|_{\mathbf{L}_{p}^{2}}+K\|\nabla w\|_{L^{4}}\|w\|_{\mathbf{L}_{p}^{2}}|\sigma|^{2} \\
& +K\|\nabla w\|_{L^{2}}|\sigma|^{2}+K\left(|\sigma|+\|w\|_{L^{\infty}}\right)\|w\|_{L^{2}}|\sigma|^{2} \\
\leq & K\left(|\sigma|+\|w\|_{\mathbf{H}_{p}^{2}}\right)\|w\|_{\mathbf{H}_{p}^{3}} \text { using Estimate (5.52). }
\end{aligned}
$$

\section{- Estimate of $G_{4}$}

We have $G_{4}=F_{4}(R(\sigma)+w)\left(\partial_{y} w, \partial_{y} w\right)$, with $F_{4}=\delta^{2} F_{3}$. So, this term is estimated as the first term of $G_{3}$ and we obtain that

$$
\left\|G_{4}\right\|_{\mathbf{L}_{p}^{2}} \leq K\left(|\sigma|+\|w\|_{\mathbf{H}_{p}^{2}}\right)\|w\|_{\mathbf{H}_{p}^{2}} \quad \text { and } \quad\left\|\nabla G_{4}\right\|_{\mathbf{L}_{p}^{2}} \leq K\left(|\sigma|+\|w\|_{\mathbf{H}_{p}^{2}}\right)\|w\|_{\mathbf{H}_{p}^{3}} .
$$

\section{- Estimate of $G_{5}$}

This term writes

$$
G_{5}=F_{5}(x, R(\sigma)+w)\left(\partial_{x} w\right)+\widetilde{F_{5}}(x, R(\sigma), w)(w)\left(\partial_{x} R(\sigma)\right),
$$


where $F_{5}$ and $\widetilde{F_{5}}$ satisfy that there exists $K$ such that for $|r| \leq \frac{1}{2}$, for $x \in \mathbb{R}$, we have:

$$
\left|F_{5}(x, r)\right|+\left|\partial_{x} F_{5}(x, r)\right| \leq K|r|, \quad\left|\partial_{r} F_{5}(x, r)\right| \leq K,
$$

and

$$
\left|\widetilde{F}_{5}(x, a, b)\right|+\left|\partial_{x} \widetilde{F}_{5}(x, a, b)\right|+\left|\partial_{r} \widetilde{F}_{5}(x, a, b)\right| \leq K .
$$

We have

$$
\begin{aligned}
\left|G_{5}\right| & \leq\left|F_{5}(x, R(\sigma)+w)\right|\left|\partial_{x} w\right|+\left|\widetilde{F_{5}}(x, R(\sigma), w)\right||w|\left|\partial_{x} R(\sigma)\right|, \\
& \leq K(|\sigma|+|w|)\left|\partial_{x} w\right|+K|\sigma||w|,
\end{aligned}
$$

thus

$$
\left\|G_{5}\right\|_{\mathbf{L}_{p}^{2}} \leq K\left(|\sigma|+\|w\|_{L^{\infty}}\right)\|w\|_{\mathbf{H}_{p}^{1}}
$$

In addition,

$$
\begin{aligned}
\left|\nabla G_{5}\right| \leq \quad & \left|\mathrm{d} F_{5}(x, R(\sigma)+w)\right|\left(\left|\partial_{x} R(\sigma)\right|+|\nabla w|\right)\left|\partial_{x} w\right|+\left|F_{5}(x, R(\sigma)+w)\right|\left|\nabla \partial_{x} w\right| \\
& +\left(\left|\partial_{x} \widetilde{F}_{5}(x, R(\sigma), w)\right|+\left|\partial_{a} \widetilde{F}_{5}(x, R(\sigma), w)\right|\left|\partial_{x} R(\sigma)\right|\right)|w|\left|\partial_{x} R(\sigma)\right| \\
& +\left|\partial_{b} \widetilde{F_{5}}(x, R(\sigma), w)\right||\nabla w||w|\left|\partial_{x} R(\sigma)\right| \\
& +\left|\widetilde{F_{5}}(x, R(\sigma), w)\right||\nabla w|\left|\partial_{x} R(\sigma)\right|+\left|\widetilde{F}_{5}(x, R(\sigma), w)\right||w|\left|\partial_{x x} R(\sigma)\right|, \\
\leq & K(|\sigma|+|\nabla w|)\left|\partial_{x} w\right|+K(|\sigma|+|w|)\left|\nabla \partial_{x} w\right|+K(1+|\sigma|)|w||\sigma| \\
& K|\nabla w||w||\sigma|+K|\nabla w||\sigma|+K|w||\sigma| .
\end{aligned}
$$

So,

$$
\begin{aligned}
\left\|\nabla G_{5}\right\|_{\mathbf{L}_{p}^{2}} & \leq K\left(|\sigma|+\|w\|_{L^{\infty}}\right)\left(\|w\|_{\mathbf{L}_{p}^{2}}+\|\nabla w\|_{\mathbf{L}_{p}^{2}}+\left\|\nabla \partial_{x} w\right\|_{\mathbf{L}_{p}^{2}}\right)+\|\nabla w\|_{L^{4}}^{2}, \\
& \leq K\left(|\sigma|+\|w\|_{\mathbf{H}_{p}^{2}}\right)\|w\|_{\mathbf{H}_{p}^{2}}
\end{aligned}
$$

- Estimate of $G_{6}$

We recall that

$$
G_{6}=F_{6}(x, R(\sigma)+w)\left(\partial_{y} w\right),
$$

with

$$
\left|F_{6}(x, r)\right|+\left|\partial_{x} F_{6}(x, r)\right| \leq K|r| \quad \text { and } \quad\left|\partial_{r} F_{6}\right| \leq K .
$$

Hence

$$
\left\|G_{6}\right\|_{\mathbf{L}_{p}^{2}} \leq\left\|F_{6}(x, R(\sigma)+w)\right\|_{L^{\infty}}\left\|\partial_{y} w\right\|_{\mathbf{L}_{p}^{2}} \leq K\left(|\sigma|+\|w\|_{L^{\infty}}\right)\|w\|_{\mathbf{H}_{p}^{1}} .
$$

In addition,

$$
\begin{aligned}
\left|\nabla G_{6}\right| \leq & \left|\partial_{x} F_{6}(x, R(\sigma)+w)\right|\left|\partial_{y} w\right|+\left|\partial_{r} F_{6}(x, R(\sigma)+w)\right|\left(\left|\partial_{x} R(\sigma)\right|+|\nabla w|\right)\left|\partial_{y} w\right| \\
& +\left|F_{6}(x, R(\sigma)+w)\right|\left|\nabla \partial_{y} w\right| \\
\leq & K(|\sigma|+|\nabla w|)\left(\left|\partial_{y} w\right|+\left|\nabla \partial_{y} w\right|\right)+K|\nabla w|\left|\partial_{y} w\right| .
\end{aligned}
$$

So

$$
\left\|\nabla G_{6}\right\|_{\mathbf{L}_{p}^{2}} \leq K\left(|\sigma|+\|w\|_{\mathbf{H}_{p}^{2}}\right)\|w\|_{\mathbf{H}_{p}^{2}} .
$$


- Estimate of $G_{7}$

This last term is given by

$$
G_{7}=\widetilde{F_{7}}(x, R(\sigma), w)(w)
$$

where, for $|a|+|b| \leq \frac{1}{2}$,

$$
\left|\widetilde{F_{7}}(x, a, b)\right|+\left|\partial_{x} \widetilde{F_{7}}(x, a, b)\right| \leq K(|a|+|b|) \quad \text { and }\left|\partial_{a} \widetilde{F_{7}}(x, a, b)\right|+\left|\partial_{b} \widetilde{F_{7}}(x, a, b)\right| \leq K \text {. }
$$

Therefore

$$
\left\|G_{7}\right\|_{\mathbf{L}_{p}^{2}} \leq\left\|\widetilde{F_{7}}(x, R(\sigma), w)\right\|_{L^{\infty}}\|w\|_{\mathbf{L}_{p}^{2}} \leq K\left(|\sigma|+\|w\|_{L^{\infty}}\right)\|w\|_{\mathbf{L}_{p}^{2}}
$$

In addition,

$$
\begin{aligned}
\left|\nabla G_{7}\right| \leq & \left|\partial_{x} \widetilde{F_{7}}(x, R(\sigma), w)\right||w|+\left|\partial_{a} \widetilde{F_{7}}(x, R(\sigma), w)\right|\left|\partial_{x} R(\sigma)\right||w| \\
& +\left|\partial_{b} \widetilde{F_{7}}(x, R(\sigma), w)\right||\nabla w||w|+\left|\widetilde{F_{7}}(x, R(\sigma), w)\right||\nabla w| \\
\leq & K(|\sigma|+|w|)|w|+K|\sigma||w|+K|\nabla w||w|+K(|\sigma|+|w|)|\nabla w| .
\end{aligned}
$$

So

$$
\left\|\nabla G_{7}\right\|_{\mathbf{L}_{p}^{2}} \leq K\left(|\sigma|+\|w\|_{L^{\infty}}\right)\|w\|_{\mathbf{H}_{p}^{1}}
$$

Therefore we obtain that

$$
\|\mathcal{G}\|_{\mathbf{L}_{p}^{2}} \leq K\left(|\sigma|+\|w\|_{\mathbf{H}_{p}^{2}}\right)\|w\|_{\mathbf{H}_{p}^{2}} \quad \text { and }\|\mathcal{G}\|_{\mathbf{H}_{p}^{1}} \leq K\left(|\sigma|+\|w\|_{\mathbf{H}_{p}^{2}}\right)\|w\|_{\mathbf{H}_{p}^{3}} .
$$

Since $\tilde{\mathcal{G}}=\left\langle\mathcal{G}_{1} \mid \frac{1}{4 \pi \cosh x}\right\rangle$, where $\mathcal{G}_{1}$ is the first component of $\mathcal{G}$, we have

$$
|\tilde{G}| \leq 2 \pi\|\mathcal{G}\|_{\mathbf{L}_{p}^{2}} \leq K\left(|\sigma|+\|w\|_{\mathbf{H}_{p}^{2}}\right)\|w\|_{\mathbf{H}_{p}^{2}}
$$

So, using that $g(\sigma)$ is bounded by below by $\frac{1}{2}$, we obtain that there exists $K$ such that if $|\sigma| \leq \eta_{0}$ and $\|w\|_{\mathbf{H}_{p}^{2}} \leq \eta_{0}$,

$$
|G| \leq K\left(|\sigma|+\|w\|_{\mathbf{H}_{p}^{2}}\right)\|w\|_{\mathbf{H}_{p}^{2}}
$$

From the expression of $\mathcal{K}_{\sigma}(w)$ we have

$$
\left|\mathcal{K}_{\sigma}(w)\right| \leq C\left(\left\|w_{2}\right\|_{\mathbf{L}_{p}^{2}}+\left\|\ell w_{1}\right\|_{\mathbf{L}_{p}^{2}}\right) .
$$

Thus, as $|a(x, \sigma)|+\left|\partial_{x} a(x, \sigma)\right| \leq K|\sigma|$, using the previously-obtained estimates we obtain that

$$
\|\mathcal{H}\|_{\mathbf{L}_{p}^{2}} \leq K\left(|\sigma|+\|w\|_{\mathbf{H}_{p}^{2}}\right)\|w\|_{\mathbf{H}_{p}^{2}}
$$

and

$$
\|\mathcal{H}\|_{\mathbf{H}_{p}^{1}} \leq K\left(|\sigma|+\|w\|_{\mathbf{H}_{p}^{2}}\right)\|w\|_{\mathbf{H}_{p}^{3}} .
$$

Concerning the right-hand side terms in Equation (4.35), from (5.61), we get:

$$
\left|\mathcal{K}_{\sigma}(w)\right| \leq K\|w\|_{\mathbf{H}_{p}^{2}},
$$

and from (5.60), since $|\sigma| \leq \eta_{0}$ and $\|w\|_{\mathbf{H}_{p}^{2}} \leq \eta_{0}$, we obtain that there exists $K$ such that

$$
|G| \leq K\|w\|_{\mathbf{H}_{p}^{2}}
$$

This concludes the proof of Proposition 4.2. 


\section{References}

[1] Allwood DA, Xiong G, Faulkner CC, Atkinson D, Petit D and Cowburn RP, Magnetic Domainwall Logic, Science 309 (2005), 1688-1692.

[2] François Alouges and Alain Soyeur, On global weak solutions for Landau-Lifshitz equations: existence and nonuniqueness, Nonlinear Anal. 18 (1992), no. 11, 1071-1084.

[3] William F. Brown, Micromagnetics, Wiley, New York (1963).

[4] Gilles Carbou, Stability of static walls for a three-dimensional model of ferromagnetic material, J. Math. Pures Appl. 93 (2010), no. 2, 183-203.

[5] Gilles Carbou, Thin Layers in Micromagnetism, Math. Models and Meth. in Applied Sciences 11 (2001), 1529-1546.

[6] Gilles Carbou and Pierre Fabrie, Time average in Micromagnetism, Journal of Differential Equations 147 (1998), 383-409.

[7] Gilles Carbou and Pierre Fabrie, Regular Solutions for Landau-Lifschitz Equation in a Bounded Domain, Differential and Integral Equations 14 (2001), 213-229.

[8] Gilles Carbou and Pierre Fabrie, Regular solutions for Landau-Lifschitz equation in $\mathbb{R}^{3}$, Commun. Appl. Anal. 5 (2001), no. 1, 17-30.

[9] Gilles Carbou and Rida Jizzini, Domain walls dynamics in a nanowire subject to an electric current, J. Differential Equations 258 (2015), no. 8, 2941-2965.

[10] Gilles Carbou and Stéphane Labbé, Stability for static walls in ferromagnetic nanowires, Discrete Contin. Dyn. Syst. Ser. B 6 (2006), no. 2, 273-290.

[11] Gilles Carbou and Sthéphane Labbé, Stabilization of Walls for Nano-Wires of Finite Length. ESAIM Control Optim. Calc. Var. 18 (2012), no. 1, 1-21.

[12] Gilles Carbou, Stéphane Labbé and Emmanuel Trélat, Control of travelling walls in a ferromagnetic nanowire, Discrete Contin. Dyn. Syst. Ser. S 1 (2008), no. 1, 51-59.

[13] Shijin Ding, Boling Guo, Junyu Lin and Ming Zeng, Global existence of weak solutions for Landau-Lifshitz-Maxwell equations, Discrete Contin. Dyn. Syst. 17 (2007), no. 4, 867-890.

[14] G. Gioia and R. D. James, Micromagnetics of very thin films, R. Soc. Lond. Proc. Ser A 453 (1997), 213-223.

[15] Boling Guo and Fengqiu Su, Global weak solution for the Landau-Lifshitz-Maxwell equation in three space dimensions, J. Math. Anal. Appl. 211 (1997), no. 1, 326-346.

[16] Todd Kapitula, On the stability of travelling waves in weighted $L^{\infty}$ spaces, J. Differential Equations 112 (1994), no. 1, 179-215.

[17] Todd Kapitula, Multidimensional stability of planar travelling waves, Trans. Amer. Math. Soc. 349 (1997), no. 1, 257-269.

[18] Stéphane Labbé, Simulation numérique du comportement hyperfréquence des matériaux ferromagnétiques. Thèse de l'Université Paris 13 (1998).

[19] Stuart S.P. Parkin, Masamitsu Hayashi, Luc Thomas, Magnetic domain-wall racetrack memory, Science 320 (2008), 190-194.

[20] Keisuke Takasao, Stability of travelling wave solutions for the Landau-Lifshitz equation, Hiroshima Math. J. 41 (2011), no. 3, 367-388. 
[21] André Thiaville and Yoshinobu Nakatani, Domain wall dynamics in nanowires and nanostrips, Spin dynamics in confined magnetic structures III, B. Hillebrands, A. Thiaville Eds. Topics in Applied Physics 101, pp. 161-206 (Springer, 2006)

[22] Augusto Visintin, On Landau Lifschitz equation for ferromagnetism, Japan Journal of Applied Mathematics 1 (1985), no. 2, 69-84.

[23] Laurence R. Walker, Bell Telephone Laboratories Memorandum, 1956 (unpublished). An account of this work is to be found in J.F. Dillon, Jr., Magnetism Vol III, edited by G.T. Rado and H. Subl, Academic, New York, 1963.

[24] Ming Yan, Christian Andreas, Attila Kákay, Felipe García-Sánchez and Riccardo Hertel, Fast domain wall dynamics in magnetic nanotubes: Suppression of Walker breakdown and Cherenkov-like spin wave emission, Appl. Phys. Lett. 99 (2011) 